\title{
ON INFINITE DIMENSIONAL TEICHMÜLLER SPACES
}

\author{
PEKKA TUKIA*
}

\section{Contents}

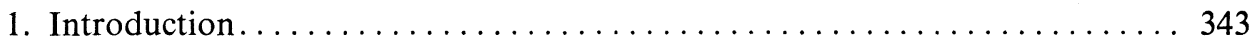

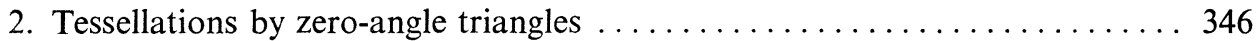

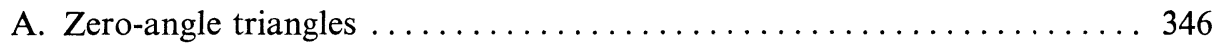

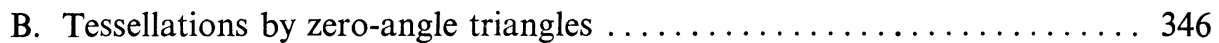

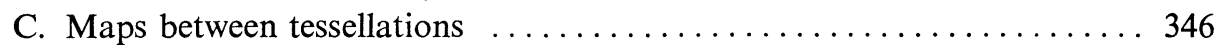

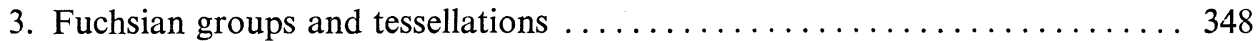

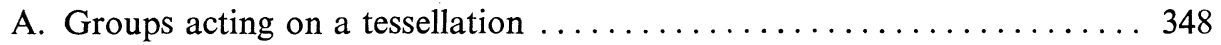

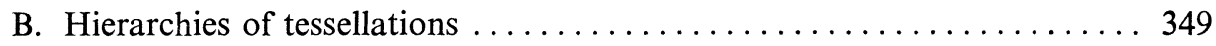

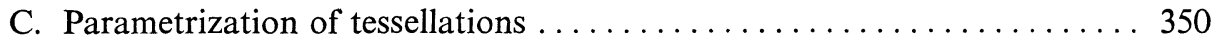

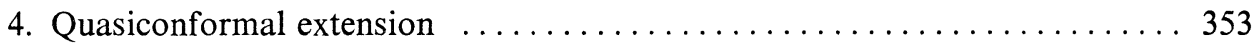

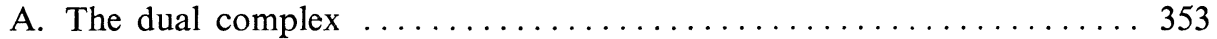

B. A canonical map between hyperbolic triangles.............. 353

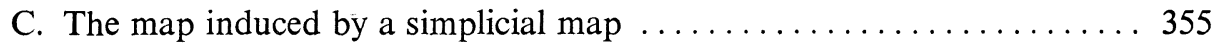

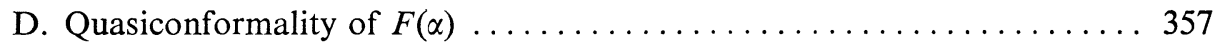

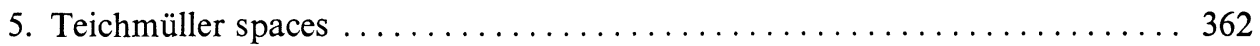

A. Parametrization of quasisymmetric maps $\ldots \ldots \ldots \ldots \ldots \ldots \ldots \ldots \ldots 2$

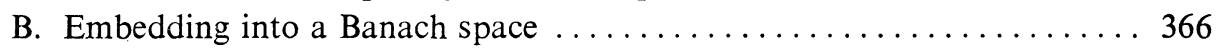

\section{Introduction}

Let $U$ be the upper half-plane $\{z \in C: \operatorname{Im} z>0\}$ and let $G$ be a Fuchsian group of $U$. We say that a homeomorphism $f: V \rightarrow W(V, W \subset \mathrm{cl} U)$ is $G$-compatible if $f \circ g \circ f^{-1}$ is the restriction of a Möbius transformation for $g \in G$. The Teichmüller space $T(G)$ of $G$ is the set of $G$-compatible quasisymmetric maps of $\boldsymbol{R}$ that fix 0 and 1 and that can be extended to a $G$-compatible quasiconformal map of $U$.

* I wish to thank the Magnus Ehrnrooth Foundation for financial support. 
A complex structure can be given to $T(G)$ as follows. A map whose domain contains 0,1 and $\infty$ is said to be normalized if it fixes 0,1 and $\infty$. Let $K(G)$ be the set of normalized $G$-compatible quasiconformal maps of cl $U$. Then $T(G)=K(G) / \sim$ where $\sim$ is the equivalence relation of $K(G)$ for which $f \sim g$ if and only if $f|\boldsymbol{R}=g| \boldsymbol{R}$. Each element $f \in K(G)$ is uniquely determined if we know its complex dilatation $\mu_{f}=\bar{\partial} f / \partial f$. Since $f$ is $G$-compatible, $\mu=\mu_{f}$ satisfies

$$
\mu(g(x))=\mu(x)\left(g^{\prime}(x) / \overline{g^{\prime}(x)}\right) \text { for all } g \in G .
$$

An element $\mu \in L^{\infty}(U, C)$ is said to be $G$-compatible if it satisfies (1.1), and the set of all $G$-compatible elements of $L^{\infty}(U, C)$ is denoted by $L^{\infty}(G)$. The (open) unit ball of $L^{\infty}(G)$ is denoted by $M(G)$. Then $f \mapsto \mu_{f}$ is a bijection $K(G) \rightarrow M(G)$.

This embedding into a complex Banach space defines a complex analytic structure on $K(G)$. The complex structure of $T(G)$ is defined as the quotient structure of $K(G) / \sim$. Bers [1, 2] (cf. also [6]) has shown that $T(G)$ with this structure is in fact a complex analytic Banach manifold. He showed that one can define a holomorphic map $\Phi: M(G) \rightarrow B(G)$, where $B(G)$ is a complex Banach space of holomorphic functions which are defined in the lower half-plane $L$, are quadratic with respect to $G$ and satisfy a norm condition. At every point $x \in M(G)$ the derivative $d \Phi(x): L^{\infty}(G) \rightarrow B(G)$ has a continuous right inverse $B(G) \rightarrow L^{\infty}(G)$. One can also show that $\Phi\left(\mu_{f}\right)=\Phi\left(\mu_{g}\right), f, g \in K(G)$, if and only if $f \sim g$. It follows that $K(G) / \sim=$ $=T(G)$ is isomorphic to $\Phi(M(G))$ which is an open subset of $B(G)$, and thus $T(G)$ is a complex analytic manifold. (Bers defines $T(G)=\Phi(M(G)$ ) in contrast to our definition $T(G)=K(G) / \sim$.) We do not make use of these results of Bers.

Earle [4] has indicated how to show that $T(G)$ is contractible if $G$ is finitely generated (cf. also [6] if $G=\{\mathrm{id}\}$ ). If, in addition, $G$ is of the first kind, then $T(G)$ is homeomorphic to a finite dimensional euclidean space. The method of proof of this result is the construction of so called "moduli" for Fuchsian groups (cf. e.g. Keen $[7,8,9])$. It has been conjectured by Bers that $T(G)$ is always contractible [1, Lecture 1].

In the present work we extend these results. It must be assumed that the group $G$ satisfies certain regularity conditions. These conditions are satisfied e.g. if $G$ is a subgroup of a finitely generated group $H$ such that $U / H$ is non-compact. Proposition 3.3 (e) implies that if $G$ is an arbitrary group such that $U / G$ is non-compact, then there is a homeomorphism $f$ of $\operatorname{cl} U$ such that $f \circ G \circ f^{-1}$ is a Fuchsian group satisfying these conditions. Under these circumstances we show that $T(G)$ is contractible (Theorem 5.2). We show this by constructing for every quasisymmetric $G$-compatible map of $\boldsymbol{R}$ a quasiconformal $G$-compatible extension to $U$. This extension defines a real analytic section $s: T(G) \rightarrow M(G)$, i.e. $s$ is a right inverse to the quotient map $M(G) \rightarrow T(G)$. Since $M(G)$ is contractible, this implies the contractibility of $T(G)$. Earle [5] has also shown that in case $G=\{\mathrm{id}\}$ the Beurling-Ahlfors extension of a quasisymmetric map defines a real analytic section $T(1) \rightarrow M(1)$. 
Our method of extension is in some respects similar to Carleson's piecewise linear extension [3].

We also show that if $G$ satisfies certain additional conditions, then $T(G)$ is homeomorphic to a Banach space $E$ and as a real analytic Banach manifold $T(G)$ is equivalent to an open and convex subset of $E$ (Theorem 5.5). This is true e.g. if $G$ is contained in a finitely generated group of the second kind. It turns out that $E$ is isomorphic to $l^{\infty}$ as a real Banach space, where $l^{\infty}$ is the Banach space of bounded sequences $\left(k_{i}\right), i \geqq 0$, with supremum norm. Thus it might seem that the contrast between $M(G)$ and $T(G)=M(G) / \sim$ lies in the fact that $T(G)$ is a $l^{\infty}$-manifold whereas $M(G)$ is a $L^{\infty}$-manifold. But there is no contrast since $l^{\infty}$ and $L^{\infty}$ are isomorphic (Pełcszyński [12]). Thus also the space $B(G)$ of holomorphic maps of $L$ is isomorphic to $l^{\infty}$ as a real Banach space, since an open subset of $B(G)$ (i.e. $\Phi(M(G))$ ) can be mapped by a real analytic isomorphism to an open subset of $E$.

As we have already indicated we show that under these circumstances any $G$-compatible quasisymmetric map $f$ admits a $G$-compatible quasiconformal extension to $U$. For arbitrary $G$, the existence of such an extension is not known but Lehto [11] has shown that if the quasisymmetry of $f$ is close to 1 , such an extension always exists.

This and other results of the paper [11] by Lehto will be discussed in the remark at the end of Section 4.

Our method is to find for $G$ (we consider only the case with $U / G$ non-compact) a special tessellation $\mathscr{T}$ of $U$ which is invariant under $G$ and whose triangles are hyperbolic triangles of $U$ with two or three zero angles. If such a triangle $T$ has a vertex $v$ at which the angle of $T$ is not zero, then $v$ is the fixpoint of some elliptic element of $G$. Each map $f \in T(G)$ determines automatically a tessellation $\mathscr{T}_{f}$ which is invariant under $f \circ G \circ f^{-1}$. It turns out that we can parametrize such tessellations $\mathscr{T}_{f}$ by sequences $\left(k_{i}^{f}\right), i \in I$, of real numbers, where the index set $I$ is the set of sides of $\mathscr{T}$ factored by $G$. We use these numbers $\left(k_{i}^{f}\right)$ to determine the Teichmüller space of $G$. Thus our method is a development of the idea of "moduli" in the finite dimensional case and also an extension of [15], where we proved similar results for $G=\{$ id $\}$.

Our results are known if $T(G)$ is finite dimensional. Therefore we have emphasized infinite dimensionality in the title, although our methods apply also in the finite dimensional case if $U / G$ is non-compact (cf. Remark 2 in Section 5).

This work was motivated by the results of [14, Proposition p. 15] and [13, Case 2 in the proof of Theorem 3.6], where we proved that any Fuchsian group $G$ such that $U / G$ is non-compact has a fundamental domain $D$ such that $\operatorname{bd}_{U} D$ consists of a family of hyperbolic lines and rays that do not intersect except possibly at the fixpoints of elliptic elements of $G$. The desire to utilize fundamental domains of this kind to obtain moduli for infinitely generated Fuchsian groups was the main motive in this work. (Fundamental polygons for infinitely generated Fuchsian groups have been constructed also by Keen [10]. Unlike ours, the sides of her fundamental polygons can intersect at points that are not fixpoints of elliptic elements of $G$.) 
We use $\mathrm{cl}$ and bd to denote closure and boundary. Sometimes it is not clear where closure or boundary is taken. Then we clarify the situation by denoting $\mathrm{cl}_{A} B$, bd $_{A} B$ or by denoting $\mathrm{cl} B$ (closure in $A$ ). We use also cl to denote class. If a group $G$ acts on a set $A$, the orbit $G a, a \in A$, is denoted by cl $a$ (=class of $a$ ). We may again for clarity denote $\mathrm{cl}_{G} a$ or $\mathrm{cl} a$ (in $A / G$ ).

\section{Tessellations by zero-angle triangles}

A. Zero-angle triangles. A (proper) zero-angle triangle of the open upper halfplane $U$ is a degenerated triangle $T \subset U$ such that $T$ is closed in $U$ and that $\operatorname{bd}_{U} T$ consists of three hyperbolic lines, not intersecting in $U$, but, if extended to the boundary $\partial U=\boldsymbol{R} \cup\{\infty\}$, intersecting at three points of $\partial U$. The hyperbolic lines bounding $T$ are the sides of $T$, and the three points of $\operatorname{cl} T \backslash T$ in $R \cup\{\infty\}$ are the vertices of $T$. If $T^{\prime}$ is another such triangle, then there is a unique, conformal or anticonformal, Möbius transformation of $U$ carrying $T$ to $T^{\prime}$ in such a way that the vertices of $T$ are carried to pre-assigned vertices of $T^{\prime}$.

If we were interested only in such Fuchsian groups that do not contain elliptic elements, it would be sufficient to consider only zero-angle triangles of the above kind. However, if we consider groups with elliptic elements, we must consider also triangles $T$ such that $\partial T$ consists of two hyperbolic rays $R_{1}$ and $R_{2}$ with one common point $x_{0} \in U$ and a hyperbolic line $L$, not intersecting $R_{1}$ nor $R_{2}$ in $U$, but such that cl $L \cap \mathrm{cl} R_{1}=\left\{x_{1}\right\}$ and $\mathrm{cl} L \cap \mathrm{cl} R_{2}=\left\{x_{2}\right\}$. The points $x_{0}, x_{1}$ and $x_{2}$ are the vertices of $T$ and $L, R_{1}$ and $R_{2}$ are its sides. The point $x_{0}$ is the non-zero vertex of $T$, others are zero-angle vertices of $T$.

B. Tessellations by zero-angle triangles. Let $V$ be an open, connected subset of $U$. A tessellation of $V$ by zero-angle triangles is a collection $\mathscr{T}$ of zero-angle triangles of $U$ such that:

(i) $V=\bigcup_{T \in \mathscr{T}} T$.

(ii) If $x \in V$, the set $\{T \in \mathscr{T}: x \in T\}$ is finite.

(iii) If $T, S \in \mathscr{T}, T \neq S$, then $T \cap S=\emptyset$ or a common side or a common vertex.

In most cases $V=U$ and it is easy to see that $U$ has such tessellations. E.g., let $T_{0}$ be the zero-angle triangle with vertices 0,1 and $\infty$ and let $G_{0}$ be the group whose generators are reflections in the sides of $T_{0}$. Let $\mathscr{T}_{0}$ be the tessellation $\left\{g\left(T_{0}\right): g \in G_{0}\right\}$ of $U$. A vertex or a side of $\mathscr{T}$ is a vertex or a side of some triangle of $\mathscr{T}$. We denote by $\mathscr{T}^{0}$ the set of all vertices of $\mathscr{T}$, by $\mathscr{T}^{1}$ the set of all sides of $\mathscr{T}$ and by $\mathscr{T}^{2}=\mathscr{T}$ the set of all triangles of $\mathscr{T}$. In the following a tessellation will always mean a tessellation of $V \subset U$ satisfying the above conditions. If $V \neq U$, then $\operatorname{bd}_{U} V$ consists of a number of disjoint hyperbolic lines.

C. Maps between tessellations. Let $\mathscr{T}$ and $\mathscr{S}$ be two tessellations. A simplicial map $\alpha: \mathscr{T} \rightarrow \mathscr{S}$ is a bijection $\mathscr{T}^{0} \cup \mathscr{T}^{1} \cup \mathscr{T}^{2} \rightarrow \mathscr{S}^{0} \cup \mathscr{S}^{1} \cup \mathscr{S}^{2}$ such that it and its 
inverse preserve the side and the vertex relations. Since a vertex $v \in T \in \mathscr{T}$ is a non-zero vertex if and only if $v$ is a vertex in a finite number of triangles of $\mathscr{T}$, non-zero vertices are mapped onto non-zero vertices. A simplicial map $\mathscr{T} \rightarrow \mathscr{S}$ is uniquely determined if we know how it maps the vertices of a given triangle of $\mathscr{T}$.

A geometric map $f: \mathscr{T} \rightarrow \mathscr{S}$ is a homeomorphism of $V=\bigcup_{T \in \mathscr{T}} T \rightarrow W=\bigcup_{T \in \mathscr{S}} T$ such that $T$ is a triangle of $\mathscr{T}$ if and only if $f(T)$ is a triangle of $\mathscr{S}$. A homeomorphism $f$ is a geometric realization of a simplicial map $\alpha$ if $f(T)=\alpha(T)$ for $T \in \mathscr{T}$. It is clear that every simplicial map has a geometric realization and, conversely, every geometric map is a geometric realization of a simplicial map.

We assume now that $V=W=U$. Under these conditions a geometric map $f: \mathscr{T} \rightarrow \mathscr{S}$ has always an extension to a homeomorphism of cl $U$. (If $x \in \partial U \backslash \mathscr{T}^{0}$, notice that $x$ has a basis of neighbourhoods $U_{i}, i>0$, such that $\operatorname{bd}_{U}\left(U_{i} \backslash \partial U\right)$ is a side of some triangle of $\mathscr{T}$. The extension to $\mathscr{T}^{0}$ is also obvious.) Conversely, consider a tessellation $\mathscr{T}$ of $U$ and a homeomorphism $f$ of $\partial U$. We can define a tessellation $\mathscr{T}_{f}$ of $U$ as follows: If $v \in \mathscr{T}^{0}$ is a non-zero vertex, let $U_{v}=\cup\{T: T \in \mathscr{T}$ and $v \in T\}$. If $s_{1}, \ldots, s_{n}$ are the components of $\mathrm{bd}_{U} U_{v}$, then each $s_{i}$ is a hyperbolic line and $s_{i} \in \mathscr{T}^{1}$. Let $s_{i}^{\prime}$ be the hyperbolic line with endpoints $f(x)$ and $f(y)$ if $x$ and $y$ are the endpoints of $s_{i}$ in $\partial U$. Then there is a well-defined closed subset $U_{v}^{\prime}$ of $U$ such that $\operatorname{bd}_{U} U_{v}^{\prime}=s_{1}^{\prime} \cup \ldots \cup s_{n}^{\prime}$. Let $v_{f}$ be some point of int $_{U} U_{v}^{\prime}$. If $v \in \mathscr{T}^{0}$ is a zero-angle vertex, let $v_{f}=f(v)$. If $s \in \mathscr{T}^{1}$, let $s_{f}$ be the hyperbolic ray or line with endpoints $v_{f}$ and $u_{f}$, where $u$ and $v$ are the endpoints of $s$. Finally, if $T$ is a triangle of $\mathscr{T}$ with sides $s_{i}$, $i \leqq 3$, let $T_{f}$ be the unique zero-angle triangle with sides $\left(s_{i}\right)_{f}, i \leqq 3$. Then $\mathscr{T}_{f}=\left\{T_{f}: T \in \mathscr{T}\right\}$ is a tessellation of $U$ and $x \mapsto x_{f}, x \in \mathscr{T}^{0} \cup \mathscr{T}^{1} \cup \mathscr{T}^{2}$, is a simplicial map $\alpha_{f}: \mathscr{T} \rightarrow \mathscr{T}_{f}$. Any map that is a geometric realization of $\alpha_{f}$ has extension to $\partial U$ coinciding with $f$.

In the above definition of $\mathscr{T}_{f}$ we had a certain freedom in the choice of the nonzero vertices of $\mathscr{T}_{f}$. If there are no such vertices in $\mathscr{T}$, the tessellation $\mathscr{T}_{f}$ is uniquely determined. Later we will consider Fuchsian groups acting on a tessellation, and we require that each non-zero vertex is the fixpoint of some elliptic transformation in the group $G$ we are considering. This requirement makes $v_{f}$ again unique for nonzero vertices $v$.

Let $\mathscr{T}$ be some tessellation with a triangle $T$ that has three zero-angle vertices. Then, by performing a suitable Möbius transformation of $U$, it is always possible to assume that the vertices of $T$ are 0,1 and $\infty$. If this is the case, we say that $\mathscr{T}$ is normalized. A normalized simplicial map $\alpha: \mathscr{T} \rightarrow \mathscr{P}$ between two normalized tessellations is a map that maps the vertices 0,1 and $\infty$ of $\mathscr{T}$ to the vertices 0,1 and $\infty$ of $\mathscr{S}$ (in this order). A normalized simplicial map is unique; it depends only on $\mathscr{T}$ and $\mathscr{S}$.

It should be noticed that a tessellation can be normalized only if it contains a triangle with three zero-angle vertices; not all tessellations contain such triangles. However, in later applications in all cases of interest it is possible to normalize a tessellation. 


\section{Fuchsian groups and tessellations}

A. Groups acting on a tessellation. Let $G$ be a group of Möbius transformations of $U$. We say that $G$ acts on a tessellation $\mathscr{T}$ if $g(T) \in \mathscr{T}$ whenever $T \in \mathscr{T}$ for each $g \in G$. If $G$ acts on a tessellation, then $G$ necessarily acts discontinuously in $U$. Hence $G$ is a Fuchsian group of $U$ if all its elements are orientation preserving. While it appears that our results are true also for groups containing orientation reversing elements, we nevertheless restrict our treatment to Fuchsian groups. By a Fuchsian group we always mean a Fuchsian group of $U$.

If a Fuchsian group $G$ acts on a tessellation $\mathscr{T}$, then the quotient surface $U / G$ has also a tessellation induced by $\mathscr{T}$. (The conditions (i)-(iii) must of course be modified to fit this case.) We denote this tessellation by $\mathscr{T} / G$. Let $v \in \mathrm{cl} U$. If for some $g \in G \backslash\{\mathrm{id}\}, g(v)=v$, we say that $v$ is a fixpoint of $G \backslash\{\mathrm{id}\}$ or, in short, of $G$. Let $v$ be a vertex of $\mathscr{T}$. Then we say that $v$ is an elliptic (resp. parabolic) vertex if there is an elliptic (resp. parabolic) element of $G$ with fixpoint $v$. (If $\mathscr{T}$ is a tessellation of $U$, then $v$ cannot be the fixpoint of a hyperbolic element of $G$.) This definition depends also on the group $G$ acting on $\mathscr{T}$, and if it seems that confusion could arise, we say e.g. that $v$ is an elliptic vertex of $\mathscr{T}$ with respect to $G$. If $v \in \mathscr{T}^{0}$ is elliptic or parabolic, we say also that $\mathrm{cl} v \in \mathscr{T}^{0} / G$ is elliptic or parabolic.

If a Fuchsian group $G$ acts on a tessellation, then $U / G$ cannot be compact. On the other hand, we shall show that if $U / G$ is non-compact, then there is always a tessellation of $U$ on which $G$ acts.

Let $\mathscr{T}$ be a tessellation. We say that $\mathscr{T}$ is a normal tessellation relative to $G$ if $G$ acts on $\mathscr{T}$ and if each $x \in U$ that is the fixpoint of an elliptic element $g \in G$ of order $\geqq 3$ is a vertex of $\mathscr{T}$, and if all the triangles of $\mathscr{T}$ with a common non-zero vertex are equivalent under $G$. (Note that if $x \in U$ is the fixpoint of an elliptic element of $G$ order at most 2, then $x$ lies on some side of $\mathscr{T}$.)

Proposition 3.1. Let $G$ be a Fuchsian group of $U$ such that $U / G$ is non-compact. Then there is a tessellation of $U$ that is normal relative to $G$.

Proof. This follows essentially from results in [13] and [14]. For according to [14, Proposition p. 15] $G$ has a fundamental domain $D$ such that $\operatorname{bd}_{U} D=\bigcup_{i \in I} T_{i}$, where each $T_{i}$ is a hyperbolic line or a hyperbolic ray and (a) for each $i \in I$, there is $g_{i} \in G$ such that $g_{i}\left(T_{i}\right)=T_{j}$ for some $j \neq i, j \in I$ (in which case $g_{j}\left(T_{j}\right)=T_{i}$ ), (b) $T_{i} \cap T_{j}=\emptyset$ if $i, j \in I, i \neq j$ except if $T_{i}$ is a ray and $T_{j}=g_{i}\left(T_{i}\right)$. In case (b) $T_{i} \cap T_{j}$ is the common endpoint of the rays $T_{i}$ and $T_{j}$; it is also the fixpoint of the elliptic transformation $g_{i}$. (Cf. also [13, Case 2 in the proof of Theorem 3.6 pp. 34-35], where this was proved for groups of the first kind.)

Now it is easy to add hyperbolic lines to $D$ in such a way that a tessellation of $D$ by zero-angle triangles results. If we take all transforms of these triangles by elements of $G$ we get a tessellation $\mathscr{T}$ of $U$ on which $G$ acts.

Note that if $x \in U$ is the fixpoint of some elliptic element $g$ of $G$, then $x$ is equi- 
valent to some point $x^{\prime} \in \mathrm{bd}_{U} D$. Therefore $\left\{x^{\prime}\right\}=T_{i} \cap T_{j} \quad\left(T_{i}\right.$ and $T_{j}$ as in (b)), Thus if the order of $g_{i} \geqq 3$, then $x$ is a non-zero vertex. If the order of $g_{i}$ equals 2 . then $T_{i} \cup T_{j}$ is a hyperbolic line, and in this case $x$ lies on some side of $\mathscr{T}$.

Remarks. Let us note that if $T \in \mathscr{T}$ has a non-zero vertex, then there is a triangle $T^{\prime} \in \mathscr{T}$ with three zero-angle vertices such that $T \cap T^{\prime}$ is a side of $\mathscr{T}$, except if $U^{\prime} / G$ is a triply punctured sphere where $U^{\prime}$ is $U$ from which fixpoints of elliptic elements have been removed. Thus $\mathscr{T}$ has triangles with three zero-angles, the above case excepted.

Also, if $G$ acts on $\mathscr{T}$ and $x \in U$ is a fixpoint of an elliptic element $g \in G$ of order $n=3$, then $x$ need not be a non-zero vertex of $\mathscr{T}$ since it may be that $x \in$ int $_{U} T$ for some $T \in \mathscr{T}$. However, if $n>3$, then $x$ is always a non-zero vertex, and even if $n=3$, there is always a subdivision $\mathscr{T}^{\prime}$ of $\mathscr{T}$ such that $x$ is a non-zero vertex of $\mathscr{T}^{\prime}$.

Let $f: U \rightarrow U$ be a homeomorphism and $G$ some Fuchsian group of $U$. Then we say that $f$ is G-compatible if $f \circ g \circ f^{-1}$ is a Möbius transformation of $U$ for each $g \in G$. This means that $f$ defines a homeomorphism $U / G \rightarrow U / G^{\prime}$ with $G^{\prime}=$ $=f \circ G \circ f^{-1}$. In the same manner, if $h$ is a homeomorphism of $\partial U$, we say that $h$ is $G$-compatible if $h \circ g \circ h^{-1}$ is the restriction of a Möbius transformation of $C \cup\{\infty\}$. A simplicial map $\alpha: \mathscr{T} \rightarrow \mathscr{S}$ (where $G$ acts on $\mathscr{T}$ ) is $G$-compatible if $\alpha \circ g \circ \alpha^{-1}$ (where we regard $g$ as a simplicial map of $\mathscr{T}$ ) is a simplicial map of $\mathscr{S}$ admitting a geometric realization which is a Möbius transformation of $U$.

B. Hierarchies of tessellations. Later, when we construct quasiconformal extensions to $U$ of quasisymmetric maps, we need an additional structure on tessellations. We call this a hierarchy of a tessellation $\mathscr{T}$. Although it cannot be motivated in the present context, we define here the hierarchy of a tessellation relative to a Fuchsian group $G$ acting on $\mathscr{T}$ and prove the existence of such a hierarchy.

Let $T, T_{1}, \ldots, T_{n}$ be zero-angle triangles of $U$ with a common vertex $v \in \partial U$ and assume that $T$ has three zero-angle vertices. We say that $T$ groups together triangles $T_{1}, \ldots, T_{n}$ at $v$ if there is a neighbourhood $V$ of $v$ such that

$$
T \cap V=\left(T_{1} \cup \ldots \cup T_{n}\right) \cap V
$$

and that $T_{i} \cap T_{j}=\mathrm{a}$ common side if $|i-j|=1$ and $=\emptyset$ if $|i-j|>1$. A hierarchy of triangles of a tessellation $\mathscr{T}$ (or, in short, a hierarchy of $\mathscr{T}$ ) is an (indexed) collection

$$
\mathscr{H}=\left\{T_{i j}(v): i \geqq 0, j \in \boldsymbol{Z}, v \text { is a zero-angle vertex of } \mathscr{T}\right\}
$$

of zero-angle triangles of $U$ that satisfy conditions (a)-(e) below. We say that a triangle $T_{i j}(v)$ of $\mathscr{H}$ is a triangle of level $i$. In general, $T_{i j}(v) \in \mathscr{T}$ only if $i=0$ (cf. (c)).

(a) If $v$ is a zero-angle vertex of $\mathscr{T}$, then $\left\{T_{0 j}(v): j \in \boldsymbol{Z}\right\}$ is the set of triangles of $\mathscr{T}$ with vertex $v$.

(b) Each triangle of $\mathscr{T}$ of level $i>0$ has three zero angles. 
(c) Each triangle $T_{i j}(v)$ of $\mathscr{H}$ groups together $n$ triangles of $\left\{T_{i-1, j}(v): j \in \boldsymbol{Z}\right\}$, $i>0$. If $i>1$, then $n=2$; if $i=1$, then $n>0$ may vary but is bounded by a constant $N_{0} \in N$.

(d) Let $s_{i j}(v)=T_{i, j-1}(v) \cap T_{i j}(v) \in \mathscr{T}^{1}$ if $i, j \in \boldsymbol{Z}, i \geqq 0$ and $v \in \mathscr{T}^{0} \cap \partial U$. If $s_{i j}(v)$ is a hyperbolic line, let $x_{i j}(v)$ be the other endpoint of $s_{i j}(v)(\neq v)$; if $s_{i j}(v)$ is a ray, let $x_{i j}(v) \in \partial U$ be such that the hyperbolic line with endpoints $v$ and $x_{i j}(v)$ contains $s_{i j}(v)$ as a subset. Then the sequence

$$
\ldots, x_{i,-1}(v), x_{i 0}(v), x_{i 1}(v), \ldots
$$

is an increasing sequence of points of $\partial U \backslash\{v\}$ in the natural order of $\partial U \backslash\{v\}$ (induced by the positive orientation of $\partial U$ ).

(e) $s_{i 0}(v)=s_{00}(v)$ for $i \geqq 0$ and $v \in \mathscr{T}^{0} \cap \partial U$.

We then suppose that a Fuchsian group $G$ acts on $\mathscr{T}$. The hierarchy $\mathscr{H}$ is invariant under $G$ if, for $i \geqq 0, j \in \boldsymbol{Z}$ and $v \in \mathscr{T}^{0} \cap \partial U$,

$$
g\left(T_{i j}(v)\right)=T_{i j}(g(v)) \text { if } \quad i, j \in \boldsymbol{Z}, i \geqq 0, v \in \mathscr{T}^{0} \cap \partial U
$$

and $g \in G$ with $g(v) \neq v$. If $g(v)=v$, let $n>0$ be the number of oriented sides of $\mathscr{T}^{1} / G$ with $\mathrm{cl}_{G} v$ as the positive endpoint. Let $T_{i j}(v)$ group together $k_{i j}$ triangles of level 0 . We assume that there is $i^{\prime} \geqq 0$ such that $k_{i j}=k_{i} n$ for $i \geqq i^{\prime}$ with $k_{i} \geqq 1$ and $k_{i^{\prime}}=1$. If $i \leqq i^{\prime}$, we assume that, for some $l=l_{i} \in \mathbf{Z}$,

$$
g\left(T_{i j}(v)\right)=T_{i, j+l}(v)
$$

Proposition 3.2. Let $G$ be a Fuchsian group acting on a tessellation $\mathscr{T}$. Then there is a hierarchy $\mathscr{H}$ of $\mathscr{T}$ which is invariant under $G$.

Proof. Let $F$ contain exactly one point of each orbit $G v, v \in \mathscr{T}^{0} \cap \partial U$. We can construct $\left\{T_{i j}(v): i \geqq 0, j \in \boldsymbol{Z}\right\}$ freely for $v \in F$; after that the hierarchy is fixed by (3.1). This is easily done. We simply choose $s_{00}(v)$ and, if $v$ is not a fixpoint for elements of $G \backslash\{$ id $\}$, the set $\left\{T_{i j}(v): i \geqq 0, j \in \boldsymbol{Z}\right\}$ is fixed if each triangle of level $i$ groups together exactly two triangles of level $i-1$. If $v$ is a fixpoint for some $g \in G \backslash\{$ id $\}$, there is some trouble due to the fact that the number of oriented sides of $\mathscr{T}^{1} / G$ with $\mathrm{cl}_{G} v$ as the positive endpoint need not be a power of 2 . However, in this case we can arrange that each triangle of level 1 groups together two or three triangles of level 0 , and each triangle of level $i>1$ groups together exactly two triangles of level $i-1$.

C. Parametrization of tessellations. Let $\mathscr{T}$ be some normalized tessellation. We describe a method by which one can parametrize all normalized tessellations that are simplicially isomorphic to $\mathscr{T}$ by means of a normalized simplicial map $\alpha$ (note that $\alpha$ is uniquely determined). 
Let $T$ and $T^{\prime}$ be two zero-angle triangles such that $T \cap T^{\prime}=s$, a common side that is a hyperbolic line. Let $v$ (resp. $v^{\prime}$ ) be the vertex of $T$ (resp. $T^{\prime}$ ) that is not on $s$. Let $L$ be the hyperbolic line such that $v, v^{\prime} \in \mathrm{cl} L$ (closure in $\mathrm{cl} U$ ). If $L$ is orthogonal to $s$, we say that $T$ and $T^{\prime}$ are symmetric with respect to $s$. We define a number $k\left(T, T^{\prime}\right)$ that measures the lack of symmetry of $T$ and $T^{\prime}$ with respect to $s$. We choose an orientation for $s$ and let the orientations of $T$ and $T^{\prime}$ be induced from that of $U$. Then one of the triangles $T$ and $T^{\prime}$, say $T^{\prime}$, is oriented compatibly with $s$, the other, $T$, is not. Thus there is a well-defined Möbius transformation $g$ of $U$ which fixes the endpoints of $s$ and for which $g\left(T^{\prime}\right)$ and $T$ are symmetric. If we transform the positive endpoint of $s$ to $\infty$ and the other endpoint to 0 , then $g$ is of the form $g(z)=\lambda z, z \in U$, where $\lambda>0$. We define $k\left(T, T^{\prime}\right)=\lambda$. We call $k\left(T, T^{\prime}\right)$ the glide coefficient of $s$ (with respect to $T$ and $T^{\prime}$ ). Then $\log k\left(T, T^{\prime}\right.$ ) is the hyperbolic length that $T^{\prime}$ must glide along $s$ in order that $T^{\prime}$ and $T$ are in a symmetric position. Note that $k\left(T, T^{\prime}\right)$ does not depend on the chosen orientation of $s$.

In case $T \cap T^{\prime}=s$ is a hyperbolic ray, we say that $T$ and $T^{\prime}$ are symmetric (with respect to $s$ ) if $T^{\prime}$ coincides with the image of $T$ under the reflection on $s$. Also in this case we could define numbers that measure the lack of symmetry of $T$ and $T^{\prime}$ but we do not need them. If any two adjacent triangles of a tessellation $\mathscr{T}$ are symmetric, we say that $\mathscr{T}$ is symmetric. If $v$ is a vertex of $\mathscr{T}$ and any two adjacent triangles of $\mathscr{T}$ with vertex $v$ are symmetric, we say that $\mathscr{T}$ is symmetric at $v$.

Let $\mathscr{T}$ and $\mathscr{T}^{\prime}$ be two normalized tessellations and $\alpha: \mathscr{T} \rightarrow \mathscr{T}^{\prime}$ a normalized simplicial map. Then $\alpha$ is uniquely determined. Thus there is a one-to-one correspondence between normalized simplicial maps $\alpha$ of $\mathscr{T}$ and the image $\alpha(\mathscr{T})$. We can associate with $\mathscr{T}^{\prime}$ (or with $\alpha$ ) a sequence $k_{s}, s \in \mathscr{T}^{1}$ and $s$ is a hyperbolic line. Set $k_{s}=k\left(\alpha(T), \alpha\left(T^{\prime}\right)\right)$, where $T$ and $T^{\prime}$ are the triangles of $\mathscr{T}$ with side $s$. We call the numbers $k_{s}$ the glide coefficients of $\mathscr{T}^{\prime}$ (or of $\alpha$ ) and denote also $k_{s}=k_{s}(\alpha)$. Given the numbers $k_{s}$, the tessellation $\mathscr{T}^{\prime}$ (like $\alpha$ ) is uniquely determined if $\mathscr{T}^{\prime}$ is symmetric at non-zero vertices and given such a sequence $k_{s}$ of positive real numbers, there is a unique normalized simplicial map $\alpha: \mathscr{T} \rightarrow \mathscr{T}^{\prime}$ such that $k_{s}=k_{s}(\alpha)$ if $s \in \mathscr{T}^{1}$ is a hyperbolic line and $\mathscr{T}^{\prime}$ is symmetric at non-zero vertices.

We assume then that a Fuchsian group $G$ acts on $\mathscr{T}$. If $\alpha: \mathscr{T} \rightarrow \mathscr{T}^{\prime}$ is $G$-compatible, we must have

$$
k_{s}(\alpha)=k_{g(s)}(\alpha), \quad g \in G \text { and } s \in \mathscr{T}^{1} \text { is a hyperbolic line. }
$$

Conversely, if the numbers $k_{s}$ satisfy (3.3), then the normalized map $\alpha: \mathscr{T} \rightarrow \mathscr{T}^{\prime}$ is $G$-compatible. Thus all $G$-compatible normalized simplicial maps of $\mathscr{T}$ can be parametrized by sequences $\left(k_{s}\right), s \in I$, where $I=\left\{s \in \mathscr{T}^{1}: s\right.$ is a hyperbolic line $\} / G$.

Proposition 3.3. (a) A symmetric tessellation is a tessellation of $U$.

(b) Let $T$ and $T^{\prime}$ be two zero-angle triangles with three zero-angles such that $T \cap T^{\prime}$ is a common side and that both $T$ and $T^{\prime}$ group together $n$ triangles of a sym- 
metric tessellation $\mathscr{S}$ at a vertex $v \in \mathrm{cl}\left(T \cap T^{\prime}\right)$. Then

$$
\left|\log k\left(T, T^{\prime}\right)\right| \leqq M_{\mathscr{S}} \text { for some } M_{\mathscr{S}} \geqq 0 .
$$

$M_{\mathscr{S}}=0$ if $\mathscr{S}$ contains only triangles with three zero-angles.

(c) If $\beta: \mathscr{S} \rightarrow \mathscr{S}$ is a simplicial map of a symmetric tessellation $\mathscr{S}$, then $\beta$ admits a realization by a Möbius transformation.

(d) If $G$ is a Fuchsian group acting on a tessellation $\mathscr{T}$, then there is a G-compatible simplicial map $\alpha: \mathscr{T} \rightarrow \mathscr{S}$, where $\mathscr{S}$ is a symmetric tessellation.

(e) If $G$ is a Fuchsian group of $U$ such that $U / G$ is non-compact, then there is a homeomorphism of $\mathrm{cl} U$ such that $f \circ G \circ f^{-1}$ acts on a symmetric tessellation of $U$ that is normal relative to $f \circ G \circ f^{-1}$.

Proof. We have already shown (c) (eq. (3.3)). The statement (d) also is clear since obviously there is a symmetric tessellation $\mathscr{S}$ and a simplicial map $\alpha: \mathscr{T} \rightarrow \mathscr{S}$. Now $\alpha$ is $G$-compatible for any group $G$ acting on $\mathscr{T}$. Proposition 3.1, (a) and (d) imply (e) since, if $\mathscr{T}$ and $\mathscr{T}^{\prime}$ are tessellations of $U$, any geometric map $\mathscr{T} \rightarrow \mathscr{T}^{\prime}$ can be extended to a homeomorphism of $\mathrm{cl} U$. Thus it suffices to prove (a) and (b).

Let $\mathscr{S}$ be a symmetric tessellation. Let $V=\cup_{T \in \mathscr{S}} T$. To prove (a) we must show $V=U$. For this it is sufficient to show that there is $c>0$ such that if $x \in V$, then $U_{c}(x) \subset V$, where $U_{c}(x)$ is the hyperbolic open disk with center $x$ and radius $c$. If $x \in V$, let $d_{x}=\sup \left\{r: U_{r}(x) \subset V\right\}$. We will show that inf $\left\{d_{x}: x \in V\right\}>0$.

We study the situation at a vertex $v \in \mathscr{T}^{0} \cap \partial U$. It is no restriction to assume $v=\infty$. If $s \in \mathscr{S}^{1}$ has vertex $v$, let $T$ and $T^{\prime}$ be the triangles of $\mathscr{S}$ with side $s$. Since $\mathscr{S}$ is symmetric, there is a hyperbolic line $L_{s}$ orthogonal to $s$ (or to $s^{\prime}$, where $s^{\prime}$ is the hyperbolic line containing $s$ if $s$ is a ray) such that a vertex of $T$ and a vertex of $T^{\prime}$ lie on $\mathrm{cl} L_{s}$ ( $\mathrm{cl}$ in $\mathrm{cl} U$ ). Let diam denote the euclidean diameter of a subset of $\boldsymbol{C}$. Then $\operatorname{diam} L_{s}=\operatorname{diam} L_{t}=d$ if $s, t \in \mathscr{S}^{1}$ are hyperbolic lines with vertex $v=\infty$. This follows easily from the symmetry of $\mathscr{S}$ and from the fact that two successive sides of $\mathscr{S}^{1}$ with vertex $v$ cannot both be rays. Let $U_{v}=\{x+i y: x \in \boldsymbol{R}, y>d / 2\} \subset U$. The boundary of $U_{v}$ is the (euclidean) line that is tangent to those $L_{s}$, where $s \in \mathscr{S}^{1}$ has vertex $v$ and is a hyperbolic line. Then $U_{v} \subset V$ and $\inf \left\{d_{x}: x \in U_{v}\right\}>c^{\prime}>0$, where $c^{\prime}$ does not depend on $v$. Also, if $T \in \mathscr{S}^{2}$,

$$
\inf \left\{d_{x}: x \in T \text { and } x \notin U_{v} \text { for } v \in \mathscr{S}^{0} \cap \partial U\right\}>c^{\prime \prime}>0 \text {, }
$$

where $c^{\prime \prime}$ does not depend on $T$. Thus $\inf \left\{d_{x}: x \in V\right\}>0$, proving (a).

To prove (b) let $S \in \mathscr{S}^{2}$ have vertex $v=\infty$. Let the sides of $S$ with vertex $v$ be contained in the lines $s=\{x+i t: t>0\}$ and $s^{\prime}=\left\{x^{\prime}+i t: t>0\right\}$. Then the above discussion shows that $\left|x-x^{\prime}\right|$ depends only on $\max \{\alpha: \alpha$ an angle of $S\}=\beta$. The number $\left|x-x^{\prime}\right|$ is smallest when $\beta=120^{\circ}$. Thus there is $c>0$ such that $\left|x-x^{\prime}\right|>c$ regardless of $S$. This proves (b), since also $\left|x-x^{\prime}\right| \leqq d / 2$, where $d$ is as in the proof of (a). 


\section{Quasiconformal extension}

In this section we shall construct a quasiconformal extension to $U$ of a given quasisymmetric map $f$. The first idea is as follows. Let $\mathscr{T}$ be some tessellation of $U$. In Section 2.C we defined the image $\mathscr{T}_{f}$ of $\mathscr{T}$, which is also a tessellation of $U$. Now if $F: \mathscr{T} \rightarrow \mathscr{T}_{f}$ is some geometric map that coincides with $f$ at vertices $v \in \mathscr{T}^{0} \cap$ $\cap \partial U$, then $F \mid \boldsymbol{R}=f$ if $F$ is extended to cl $U$ (which can always be done). However, if we wish $F$ to be quasiconformal there are difficulties due to the fact that the triangles have zero angles. For this reason it is better to use the dual complex described below.

A. The dual complex. In the dual complex $\mathscr{T}_{d}$ of a tessellation $\mathscr{T}$, a cell of $\mathscr{T}_{d}$ corresponds to a vertex of $\mathscr{T}$, a side of $\mathscr{T}_{d}$ corresponds to a side of $\mathscr{T}$, and a vertex of $\mathscr{T}_{d}$ corresponds to a triangle of $\mathscr{T}$. Let $T$ be a zero-angle triangle with vertices $a, b$ and $c$. Let $S_{a}$ be the hyperbolic line such that $a \in \operatorname{cl} S_{a}$ (closure in $\mathrm{cl} U$ ) and that the sides of $T$ with vertex $a$ are situated symmetrically about $S_{a}$. Define similarly $S_{b}$ and $S_{c}$. Then $S_{a}, S_{b}$ and $S_{c}$ intersect at a common point $T_{d} \in$ int $_{U} T$. The point $T_{d}$ is called the middle point of $T$. If $T$ has vertices 0,1 and $\infty$, then $T_{d}=1 / 2+$ $+\left(3^{1 / 2} / 2\right) i$. The set of vertices of $\mathscr{T}_{d}$ is $\mathscr{T}_{d}^{0}=\left\{T_{d}: T \in \mathscr{T}^{2}\right\}$. If $s$ is a side of $\mathscr{T}$, let $s_{d}$ be the hyperbolic line segment joining $T_{d}$ and $S_{d}$, where $T$ and $S$ are the triangles of $\mathscr{T}$ with side $s$. The set of sides of $\mathscr{T}_{d}$ is $\mathscr{T}_{d}^{1}=\left\{s_{d}: s \in \mathscr{T}^{1}\right\}$. Finally, if $v$ is a vertex of $\mathscr{T}$, let $v_{d}$ be the closed subset of $U$ whose boundary in cl $U \backslash\{v\}$ is $\cup\left\{s_{d}: s \in \mathscr{T}^{1}\right.$ and $v$ is a vertex of $s\}$. If $v \in \mathscr{T}^{0} \cap \partial U$, the boundary of $v_{d}$ in $U$ is of the form $\ldots \cup s_{i} \cup$ $s_{i+1} \cup \ldots$, where each $s_{i} \in \mathscr{T}_{d}^{1}, i \in \boldsymbol{Z}$, and $s_{i}$ intersects only with $s_{i-1}$ and $s_{i+1}$. If we add to this union the point $v$, it is a Jordan curve. If $v \in \mathscr{T}^{0} \cap U$, then $\mathrm{bd}_{U} v_{d}$ is a Jordan curve that is a similar union $\cup s_{i}$ of a finite number of sides of $\mathscr{T}_{d}$. The set of cells of $\mathscr{T}_{d}$ is $\mathscr{T}_{d}^{2}=\left\{v_{d}: v \in \mathscr{T}^{0}\right\}$.

It is easy to see that $\mathscr{T}_{d}$ is a complex in the usual sense, i.e. the intersection of two distinct elements of $\mathscr{T}_{d}$ is either empty, a common vertex or a common side.

B. A canonical map between hyperbolic triangles. Let $T_{0}$ and $T$ be two nondegenerated hyperbolic triangles. We describe a canonical way to define a map $T_{0} \rightarrow T$. Let $A_{0}, B_{0}$ and $C_{0} \in U$ be the vertices of $T$, and $A, B$ and $C$ the vertices of $T$ such that there is an orientation preserving homeomorphism $f: T_{0} \rightarrow T$ with $f\left(A_{0}\right)=A, f\left(B_{0}\right)=B$ and $f\left(C_{0}\right)=C$. We denote by $X Y$ the hyperbolic line segment joining $X$ and $Y(\in U)$. We regard $T_{0}$ as fixed and $T$ as variable and denote the map $T_{0} \rightarrow T$ to be defined by $f=f\left(A_{0}, B_{0}, C_{0}, A, B, C\right)$ since it depends on the order of vertices.

We set

$$
f\left(A_{0}\right)=A, \quad f\left(B_{0}\right)=B \text { and } f\left(C_{0}\right)=C .
$$

Let $f \mid A_{0} B_{0}$ be the linear stretch $A_{0} B_{0} \rightarrow A B$ in the hyperbolic metric and similarly $f \mid B_{0} C_{0}$ and $f \mid C_{0} A_{0}$. If $X_{0} \in B_{0} C_{0}$, let $f \mid A_{0} X_{0}$ be the linear stretch $A_{0} X_{0} \rightarrow A X$, where $X=f\left(X_{0}\right)$. Now we have defined $f$ at all points of $T_{0}$. 
If $G$ is a subset of $C$, let $L^{\infty}(G, C)$ be the Banach space of a.e. defined, essentially bounded maps $G \rightarrow C$ with sup ess norm. Let $\mu(f)=\bar{\partial} f / \partial f$ be the complex dilatation of a quasiconformal map. We have:

Lemma 4.1. (a) The map $f\left(A_{0}, B_{0}, C_{0}, A, B, C\right)=f$ is quasiconformal. For fixed $A_{0}, B_{0}$ and $C_{0}, \mu(f)$ depends only on $x=d(A, B), y=d(B, C)$ and $z=d(C, A)$ (d the hyperbolic metric), and the map $(x, y, z) \mapsto \mu(f)$ is a real analytic map of $a$ domain of $\boldsymbol{R}^{3}$ into $L^{\infty}\left(T_{0}, \boldsymbol{C}\right)$.

(b) Let $\sum_{i, j, k \geqq 0} \mu_{i j k} u^{i} v^{j} w^{k}$ be the power series development of the map $(x, y, z) \mapsto$ $\mapsto \mu(f)$ at a point $\left(x_{1}, y_{1}, z_{1}\right)$, where each $\mu_{i j k} \in L^{\infty}\left(T_{0}, C\right)$. Then each $\left\|\mu_{i j k}\right\|_{\infty}$ depends continuously on $x_{0}=d\left(A_{0}, B_{0}\right), y_{0}=d\left(B_{0}, C_{0}\right), z_{0}=d\left(C_{0}, A_{0}\right), x_{1}, y_{1}$ and $z_{1}$ and the convergence of this series is uniform in $L^{\infty}\left(T_{0}, C\right)$ as we vary $x_{0}, y_{0}, z_{0}, x_{1}, y_{1}$ and $z_{1}$ in a compact set.

Proof. In the proof of the lemma we consider the situation in the unit disk $D$ instead of $U$. This is due to the fact that we use polar coordinates and that is simpler in $D$. We set, if $r, \vartheta \in \boldsymbol{R}$,

$$
[r, \vartheta]=r(\cos \vartheta+i \sin \vartheta)
$$

We can assume that $A_{0}=A=0, B_{0}=u_{0} \in \boldsymbol{R}^{+} \cap D, B=u \in \boldsymbol{R}^{+} \cap D, C_{0}=\left[r_{0}, \vartheta_{0}\right]$ and $C=[r, \vartheta]$ with $0<r, r_{0}<1$ and $0<\vartheta, \vartheta_{0}<\pi$. Since $(x, y, z)$ and $(u, r, \vartheta)$ are in a real analytic one-to-one correspondence, to prove (a) it suffices to show that $\mu(f)=\mu\left(f\left(A_{0}, B_{0}, C_{0}, A, B, C\right)\right) \in L^{\infty}\left(T_{0}, C\right)$ is a real analytic function of $(u, r, \vartheta)$. Indeed, the above normalization shows that $\mu(f)$ depends only on $x, y$ and $z$.

We note first that it suffices to find for each point $P \in T_{0}$ a neighbourhood $U(P)$ in $T_{0}$ such that $(x, y, z) \rightarrow \mu(f) \mid U(P)$ is a real analytic map into $L^{\infty}(U(P), C)$. This implies the lemma by compactness. It is also easy to see that if $P \in T_{0} \backslash\{0\}$, then $P$ has such a neighbourhood. Thus it suffices to consider the case $P=0$.

Let $h(s)=d(s, 0)$ for $0 \leqq s<1$, where $d$ is the hyperbolic metric of $D$. Then the function $f$ has the expression

$$
f([s, \alpha])=\left[h^{-1}(\psi(\alpha) h(s)), \varphi(\alpha)\right],
$$

where $\psi$ and $\varphi$ depend real-analytically on $u, r, \vartheta$ and $\alpha$ (but do not depend on $s$ ). It is important to note that if $\alpha=0$ or $\alpha=\vartheta_{0}$, also in this case $\varphi$ and $\psi$ have a power series expansion (in $u, r, \vartheta$ and $\alpha$ ) in a neighbourhood of $(u, r, \vartheta, \alpha)$. It is easy to see that

$$
h^{-1}(\psi(\alpha) h(s))=s \psi(\alpha) g(s, \alpha)
$$

where $g$ depends real-analytically on $u, r, \vartheta, s$ and $\alpha$, and $g$ has a power series expansion also if $s=0$ or $\alpha=0$ or $\alpha=\vartheta_{0}$, and $g(0, \alpha)=1$.

If $w \in D \backslash\{0\}$, let $e_{r}(w)=w /|w|$ and let $e_{\vartheta}(w)$ be the unit vector $\in \boldsymbol{C}$ such that $\left\{e_{\boldsymbol{r}}(w), e_{\vartheta}(w)\right\}$ is a positively oriented orthonormal basis of $\boldsymbol{C}=\boldsymbol{R}^{2}$. Now it is easy to calculate the matrix of the derivative $d f(w): \boldsymbol{R}^{2} \rightarrow \boldsymbol{R}^{2}$ if the basis of domain 
is $\left\{e_{r}(w), e_{\vartheta}(w)\right\}$ and that of range is $\left\{e_{r}(f(w)), e_{9}(f(w))\right\}$. The result is, if $w=[s, \alpha]$,

$$
\left(\begin{array}{cc}
\psi(\alpha)\left(g(s, \alpha)+s D_{1} g(s, \alpha)\right) & 0 \\
\psi^{\prime}(\alpha) g(s, \alpha)+\psi(\alpha) D_{2} g(s, \alpha) & \psi(\alpha) g(s, \alpha) \varphi^{\prime}(\alpha)
\end{array}\right) .
$$

If $s=0$, this assumes the form

$$
\left(\begin{array}{cc}
\psi(\alpha) & 0 \\
\psi^{\prime}(\alpha)+\psi(\alpha) D_{2} g(0, \alpha) & \psi(\alpha) \varphi^{\prime}(\alpha)
\end{array}\right) .
$$

Thus also if $s=0$ and $0 \leqq \alpha \leqq \vartheta_{0}, d f(w)$ is defined as the analytic extension and is non-singular. Therefore if the above matrix is interpreted as a $\boldsymbol{R}$-linear map of $\boldsymbol{C}$, its complex dilatation is defined. Since the change of basis from $\left\{e_{1}=1, e_{2}=i\right\}$ to $\left\{e_{r}(w), e_{9}(w)\right\}$ corresponds to a rotation of amount arg $w=\alpha$, we see that $\mu(f)([s, \alpha])$ can be extended for $s=0$ if $0 \leqq \alpha \leqq \vartheta_{0}$ and that it has a power series expansion (in $u, r, \vartheta, s$ and $\alpha$ ) at these points. This proves that also if $P=0$, then $P$ has a neighbourhood $U(P)$ in $T_{0}$ such that $(u, r, \vartheta) \mapsto \mu(f) \mid U(P)$ is a real analytic map into $L^{\infty}(U(P), C)$.

Similar considerations prove part (b) of the lemma.

C. The map induced by a simplicial map. Let $\mathscr{T}$ and $\mathscr{T}^{\prime}$ be tessellations by zero-angle triangles and $\alpha: \mathscr{T} \rightarrow \mathscr{T}^{\prime}$ a simplicial map. In addition we assume that $\mathscr{T}$ and $\mathscr{T}^{\prime}$ are symmetric at non-zero vertices, that a Fuchsian group $G$ (possibly trivial) acts on $\mathscr{T}$ and that $\alpha$ is $G$-compatible. Finally, we assume that $\mathscr{H}$ is a $G$-invariant hierarchy of $\mathscr{T}$. Let $\mathscr{H}^{\prime}=\left\{T_{i j}^{\prime}\left(v^{\prime}\right)\right\}$ be the hierarchy of $\mathscr{T}^{\prime}$ such that $T_{i j}^{\prime}(\alpha(v))$ has vertices $\alpha(v), \alpha(u), \alpha(w)$ if $T_{i j}(v)$ has vertices $v, u$ and $w$. This determines $\mathscr{H}^{\prime}$ uniquely. We shall denote such a hierarchy by $\alpha(\mathscr{H})$ and we set also if $i>0$

$$
\alpha\left(T_{i j}(v)\right)=T_{i j}^{\prime}(\alpha(v)) \text { for } j \in \boldsymbol{Z} \text { and } v \in \mathscr{T}^{0} .
$$

Now we describe a map $F(\alpha)$ induced by $\alpha$. The domain of definition of $F(\alpha)$ is a subdomain $V_{\mathscr{H}}$ of $V=\bigcup_{T \in \mathscr{T}} T\left(V_{\mathscr{H}}\right.$ depends on $\left.\mathscr{H}\right)$, and similarly its image is a subdomain $V_{\mathscr{H}^{\prime}}$ of $V^{\prime}=\bigcup_{T \in \mathscr{T}^{\prime}} T$ that depends on $\mathscr{H}^{\prime}$. We denote by $\mathscr{T}_{d}^{i}, i=0,1,2$, also the $i$-skeleton of $\mathscr{T}_{d}$; thus $\mathscr{T}_{d}^{i}=\cup\left\{s: s \in \mathscr{T}_{d}^{i}\right\}$. It is easy to define a map $F_{0}(\alpha): \mathscr{T}_{d}^{0} \rightarrow \check{\mathscr{T}}_{d}^{\prime 0}$. We simply set $F_{0}(\alpha)\left(T_{d}\right)=\alpha(T)_{d}$ for $T \in \mathscr{T}^{2}$. The obvious way to define $F_{1}(\alpha): \mathscr{T}_{d}^{1} \rightarrow \mathscr{T}_{d}^{\prime 1}$ is to let its restriction to a side $s_{d}, s \in \mathscr{T}^{1}$, to be the linear stretch in the hyperbolic metric in such a way that $F_{1}(\alpha)\left(s_{d}\right)=\alpha(s)_{d}$ (we assume that $\left.F_{1}(\alpha) \mid \mathscr{T}_{d}^{0}=F_{0}(\alpha)\right)$. The next step, to extend this map to a map $F(\alpha)=$ $=F_{2}(\alpha): V_{\mathscr{H}} \rightarrow V_{\mathscr{H}}$, is less obvious. We shall now do that using the hierarchy $\mathscr{H}$ of $\mathscr{T}$. In some respects our method is similar to Carleson's piecewise linear extension of a quasisymmetric mapping [3].

First we note that if $v \in \mathscr{T}^{0} \cap U$, the definition of $F(\alpha) \mid v_{d}$ is easy, since in that case our assumptions ( $\mathscr{T}$ and $\mathscr{T}^{\prime}$ are symmetric at non-zero vertices) imply that there is a Möbius transformation $g_{v}: v_{d} \rightarrow \alpha(v)_{d}$ such that $g_{v}\left|\operatorname{bd} v_{d}=F_{1}(\alpha)\right| \operatorname{bd} v_{d}$. We set $F(\alpha) \mid v_{d}=g_{v}$. 
Next, we choose a vertex $v \in \mathscr{T}^{0} \cap \partial U$, and consider the triangles $T_{i j}(v), j \in \boldsymbol{Z}$, for fixed $i \geqq 0$. As in case $i=0$ we form the middle point $T_{i j}(v)_{d}$. Let $s_{i j}(v)_{d}$ be the hyperbolic line segment joining $T_{i j}(v)_{d}$ and $T_{i, j-1}(v)_{d}$. Then $\bigcup_{j \in Z} S_{i j}(v)_{d}$ is the boundary (in $U$ ) of a closed subset $v_{d}^{i}$ of $v_{d}=v_{d}^{0}$, defined like $v_{d}$ in Section 4. A. We use a similar notation, $T_{i j}^{\prime}\left(v^{\prime}\right)$ etc., with respect to $\mathscr{T}^{\prime}$. Then it is true:

(i) Each $v_{d}^{i}$ is convex in the hyperbolic metric.

(ii) $T_{i j}(v)_{d} \in$ int $_{U} v_{d}^{i-1}, i>0$, unless $T_{i j}(v)=T_{i-1, k}(v)$ for some $k \in \boldsymbol{Z}$. (This is possible only if $i=1$, by virtue of (c) in the definition of a hierarchy.)

(iii) $v_{d}^{i} \subset v_{d}^{j}$ if $i>j$.

We define $F(\alpha)\left(T_{i j}(v)_{d}\right)=T_{i j}^{\prime}(\alpha(v))_{d}$ and let $F(\alpha) \mid s_{i j}(v)_{d}$ be the linear stretch in the hyperbolic metric such that $F(\alpha)\left(s_{i j}(v)_{d}\right)=s_{i j}^{\prime}(\alpha(v))_{d}$ if $i \geqq 0$ and $j \in \boldsymbol{Z}$.

We extend $F(\alpha)$ to each set $v_{d}^{i} \backslash v_{d}^{i+1}, i \geqq 0$. Consider the situation in Fig. 1. (We assume that $i=0$. The situation is similar, but, by (c) in Section 3.B, possibly simpler if $i>0$. We fix $j \in \boldsymbol{Z}$. Let $T_{1 j}(v)$ group together triangles $T_{0 k}(v), \ldots, T_{0, k+n}(v)$. Let $s$ be the hyperbolic line segment joining $T_{1 j}(v)_{d}$ and $T_{0 k}(v)_{d}$ and $s^{\prime}$ be the segment joining $T_{1, j+1}(v)_{d}$ and $T_{0, k+n+1}(v)_{d}$. Let $F$ be the closed subset of $\operatorname{cl}\left(v_{d}^{0} \backslash v_{d}^{1}\right)$ whose boundary in $\mathrm{cl}\left(v_{d}^{0} \backslash v_{d}^{1}\right)$ is $s \cup s^{\prime}$. We triangulate $F$ as in Fig. 1. Let the triangles of this triangulation be $T_{i}, i=0, \ldots, n+1$, such that the vertices of $T_{i}$ are $A_{i}, B_{i}$ and $C_{i}$ with $A_{i}=T_{1 j}(v)_{d}, B_{i}=T_{0, k+i}(v)_{d}, C_{i}=T_{0, k+i+1}(v)_{d}$ (if $\left.i<n+1\right)$ and $C_{n+1}=$ $T_{1, j+1}(v)_{d}$. We define similarly the triangles $T_{i}^{\prime}$ with vertices $A_{i}^{\prime}, B_{i}^{\prime}$ and $C_{i}^{\prime}$ with respect to $\mathscr{T}^{\prime}$. We set

$$
F(\alpha) \mid T_{i}=f\left(A_{i}, B_{i}, C_{i}, A_{i}^{\prime}, B_{i}^{\prime}, C_{i}^{\prime}\right),
$$

where $f(\ldots)$ is as in Lemma 4.1.

Note that if $v$ and $\alpha(v)$ are parabolic fixpoints of $G$ and $G^{\prime}=\alpha \circ G \circ \alpha^{-1}$, then there is $i \geqq 0$ such that $F(\alpha) \mid v_{d}^{i}$ is the restriction of a Möbius transformation. This concludes the definition of $F(\alpha)$. Next, we examine its properties. Obviously,

$F(\alpha)$ is G-compatible.

If $\alpha=\mathrm{id}$, we have

at the points $x$ where $F\left(\right.$ id) is defined. Let then $\mathscr{T}^{\prime \prime}$ be a tessellation and $\beta: \mathscr{T}^{\prime} \rightarrow \mathscr{T}^{\prime \prime}$ a simplicial map. We provide $\mathscr{T}^{\prime \prime}$ with the hierarchy $\beta\left(\mathscr{H}^{\prime}\right)=\beta \circ \alpha(\mathscr{H})$. If we use $\mathscr{H}^{\prime}=\alpha(\mathscr{H})$ in defining $F\left(\alpha^{-1}\right)$ and $F(\beta)$, we have

$$
\begin{aligned}
& F\left(\alpha^{-1}\right)(x)=F(\alpha)^{-1}(x) \quad \text { and } \\
& F(\beta \circ \alpha)(y)=F(\beta) \circ F(\alpha)(y)
\end{aligned}
$$

at the points $x$ where $F\left(\alpha^{-1}\right)$ and $F(\alpha)^{-1}$ are defined and at the points $y$ where $F(\alpha)$ is defined.

We examine the domain of definition of $F(\alpha)$. In this respect the following condition is decisive: 
(*) Let $v \in \mathscr{T}^{0} \cap \partial U$. Then $v$ is the only point of accumulation of $\{u: u$ is a vertex of $s \in \mathscr{T}^{1}$ such that the other endpoint of $s$ is $v$.

Condition (c) in the definition of a hierarchy guarantees:

( $\delta)$ If $v \in \mathscr{T}^{0} \cap \partial U$ satisfies (*), then $F(\alpha)$ is defined in the whole $v_{d}$.

( $\varepsilon)$ If $v^{\prime}=\alpha(v)$, $v$ as in $(\delta)$, satisfies $(*)$, then $F(\alpha)\left(v_{d}\right)=v_{d}^{\prime}$.

It is also easy to see (since $v$ has arbitrary small neighbourhoods $V$ in cl $U$ such that $\operatorname{bd}_{U} V \subset \mathscr{T}_{d}^{1}$ and similarly $\left.v^{\prime}\right)$ :

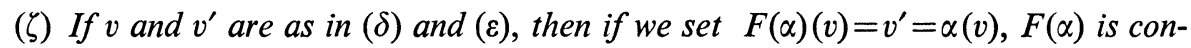
tinuous at $v$.

Finally, we note $(\eta)$ which is proved in the same manner as $(\zeta)$ :

( $\eta$ ) If $\mathscr{T}$ and $\mathscr{T}^{\prime}$ are tessellations of $U$, then $F(\alpha)$ admits an extension to a homeomorphism of $\mathrm{cl} U$ in such a way that $F(\alpha)(v)=\alpha(v)$ for $v \in \mathscr{T}^{0}$.

D. Quasiconformality of $F(\alpha)$. We study on what conditions $F(\alpha)$ is quasiconformal. In this section assumptions are unchanged; recall especially that all tessellations are assumed to be symmetric at non-zero vertices.

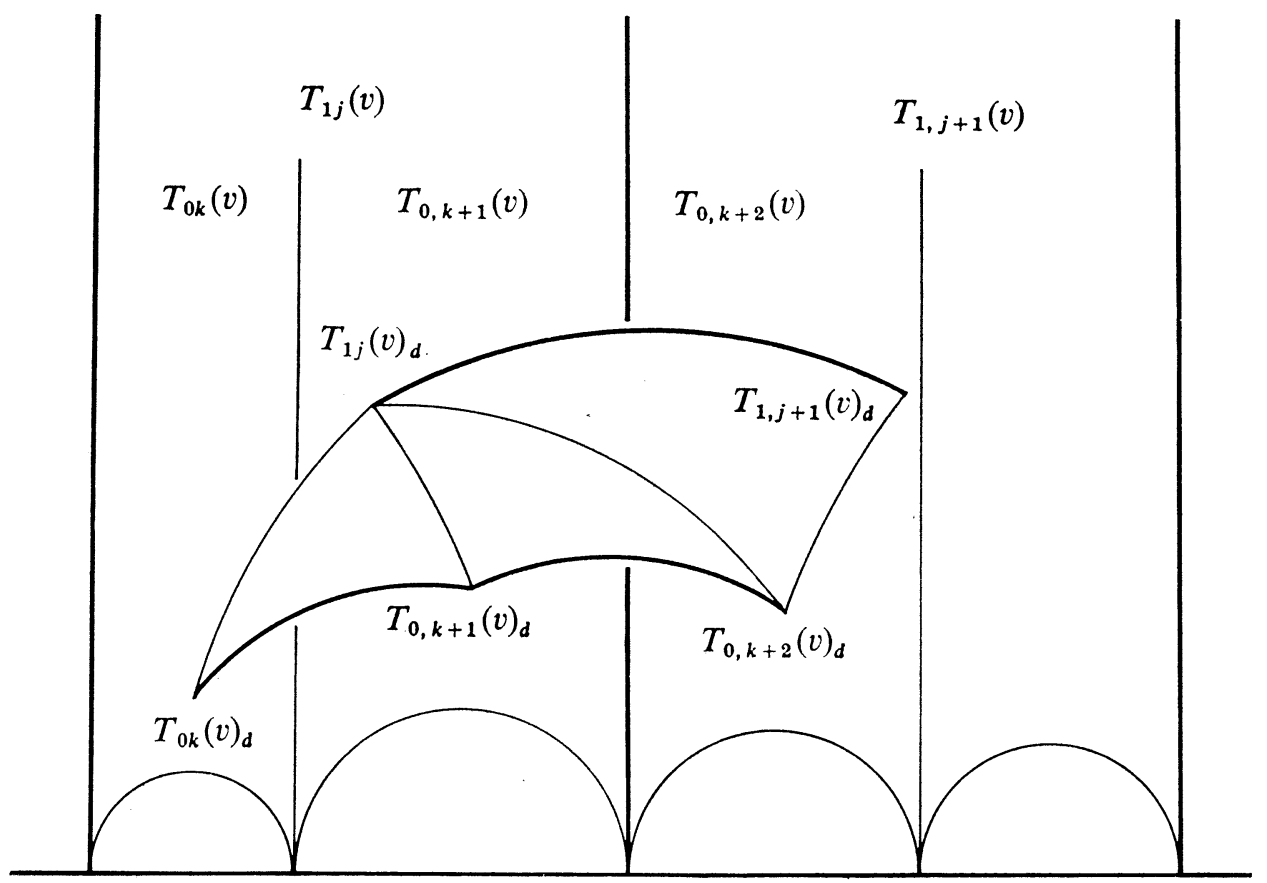

Figure 1 
For a given tessellation $\mathscr{T}$ we define numbers $k_{i j v}(\mathscr{T})$ by

$$
k_{i j v}(\mathscr{T})=k\left(T_{i, j-1}(v), T_{i j}(v)\right),
$$

where $k($,$) is the glide coefficient of Section 3.C and i \geqq 0, j \in \boldsymbol{Z}, v \in \mathscr{T}^{0} \cap \partial U$. For brevity we write $P=\boldsymbol{N} \times \boldsymbol{Z} \times\left(\mathscr{T}^{0} \cap \partial U\right.$ ) ( $N$ is the set of natural numbers, zero included) and $k_{\lambda}(\mathscr{T})$ for $k_{i j v}(\mathscr{T})$ if $\lambda=(i, j, v) \in P$. Let

$$
d(\mathscr{T})=\sup _{\lambda \in P}\left|\log k_{\lambda}(\mathscr{T})\right| .
$$

If $d(\mathscr{T})<\infty$, we say that $\mathscr{T}$ is quasisymmetric.

For a given simplicial map $\alpha: \mathscr{T} \rightarrow \mathscr{T}^{\prime}$ we define

$$
k_{i j v}(\alpha)=k_{i j \alpha(v)}\left(\mathscr{T}^{\prime}\right), \quad(i, j, v) \in P,
$$

where $\mathscr{T}^{\prime}$ is provided with the hierarchy $\mathscr{H}^{\prime}=\alpha(\mathscr{H})$. Similarly,

$$
d(\alpha)=\sup _{\lambda \in P}\left|\log k_{\dot{\lambda}}(\alpha)\right| .
$$

If $d(\alpha)<\infty$, we say that $\alpha$ is quasisymmetric.

Let $F$ be the Banach space of bounded sequences $\left(k_{\lambda}\right)_{\lambda \in P}$ with supremum norm. Let $F_{0}$ be the subset

$$
F_{0}=\left\{\left(k_{\lambda}(\alpha)\right)_{\lambda \in P}: \alpha: \mathscr{T} \rightarrow \mathscr{T}^{\prime} \text { a simplicial map with } d(\alpha)<\infty\right\} .
$$

Assume now that in addition $\mathscr{T}, \mathscr{T}^{\prime}$ and $\alpha$ are normalized. Then, given $\mathscr{T}$, since we assumed that $\mathscr{T}^{\prime}$ (like $\mathscr{T}$ ) is symmetric at non-zero vertices, $\alpha$ is uniquely determined if we know the sequence $\left(k_{\lambda}(\alpha)\right)_{\lambda \in P}$. In this case we identify $\alpha$ with the sequence $\left(k_{\lambda}(\alpha)\right)_{\lambda \in \boldsymbol{P}}$.

We can now prove if $\alpha: \mathscr{T} \rightarrow \mathscr{T}^{\prime}$ is as in Section $\mathrm{C}$ and if $\mathscr{T}$ and $\alpha$ are quasisymmetric:

Theorem 4.2. (a) $\mathscr{T}$ and $\mathscr{T}^{\prime}$ are tessellations of $U$.

(b) The map $F(\alpha)$ is a quasiconformal homeomorphism of $U$.

(c) We assume that in addition $\mathscr{T}$ and $\alpha$ are normalized. Let $H$ be a real Banach space, $H_{0} \subset H$ an open subset and $h: H_{0} \rightarrow F_{0}$ a map that is real analytic (differentiable) if regarded as a map $H_{0} \rightarrow F$. Then $x \mapsto \mu(F(h(x))$ ) is a real analytic (differentiable) map $H_{0} \rightarrow L^{\infty}(U, C)$.

Proof. We prove (a) and (b) together. Note first that we may factorize $\alpha$ as $\alpha=\beta \circ \gamma$, where $\gamma: \mathscr{T} \rightarrow \mathscr{T}_{0}$ and $\beta: \mathscr{T}_{0} \rightarrow \mathscr{T}^{\prime}$ are simplicial maps and $\mathscr{T}_{0}$ a symmetric tessellation. This follows from Proposition 3.3 (d). By Proposition 3.3 (b) $\mathscr{T}_{0}$ is quasisymmetric and therefore both $\beta$ and $\gamma$ are quasisymmetric. If the hierarchy of $\mathscr{T}_{0}$ is $\gamma(\mathscr{H})$, we have, by $(\gamma)$ of Section C, $F(\alpha)=F(\beta) \circ F(\gamma)$ and $F\left(\gamma^{-1}\right)=F(\gamma)^{-1}$. Therefore it suffices to consider the case where $\mathscr{T}$ is a symmetric tessellation. By Proposition 3.3 (a) $\mathscr{T}$ is now a tessellation of $U$. We show that $\mathscr{T}^{\prime}$ is a tessellation of $U$ and $F(\alpha)$ is quasiconformal. 
To prove the quasiconformality of $F(\alpha)$ we must have a look at Fig. 1. We defined $F(\alpha)$ piecewise in a triangulation $K$ of $U$ by (non-degenerate) hyperbolic triangles in such a way that $F(\alpha) \mid T, T \in K$, is of the form $f\left(A_{0}, \ldots, C\right)$ of Section B. Since the lengths of the sides of the triangles of $K$ as well as those of $F(\alpha)(T), T \in K$, vary in a compact set (this follows from the assumption of quasisymmetry), Lemma 4.1 implies that $\|\mu(F(\alpha))\|_{\infty}<1$, i.e. $F(\alpha)$ is quasiconformal.

Next we show that $\mathscr{T}^{\prime}$ is a tessellation of $U$. Let $v^{\prime} \in \mathscr{T}^{\prime 0} \cap \partial U$. Since $\mathscr{T}^{\prime}$ is quasisymmetric, $v^{\prime}$ satisfies (*) of Section C. Therefore $v_{d}^{\prime} \subset F(\alpha)(U)$ and $F(\alpha)(U)=$ $=V=\bigcup_{T \in \mathscr{T}} T=\bigcup_{v^{\prime} \in \mathscr{T}^{\prime} 0} v_{d}^{\prime}$. Each component $C$ of $U \backslash V$ is a closed subset of $U$ such that $\operatorname{bd}_{U} C$ is a hyperbolic line. Therefore $V$ is a Jordan domain of the Riemann sphere. Since $F(\alpha)$ is quasiconformal, it can be extended to a homeomorphism cl $U \rightarrow \operatorname{cl} V$. By $(\zeta)$ of Section $\mathrm{C}$ we must have $F(\alpha)(v)=\alpha(v)$ for $v \in \mathscr{T}^{0}$. Since $\mathscr{T}^{0}$ is dense in $\partial U, \mathscr{T}^{\prime 0}$ must also be dense in $\partial V$. This is possible only if $V=U$.

In (c) we have a family $\alpha(x): \mathscr{T} \rightarrow \mathscr{T}(x), x \in H_{0}$, of simplicial maps (set $h(x)=$ $=\left(k_{\lambda}(\alpha(x))_{\lambda \in P}\right)$. Since the lengths of the sides of the triangles in Fig. 1 for $\alpha(x)$ are real analytic (differentiable) functions of $x \in H_{0}$ that vary in a compact set, the result follows by Lemma 4.1 .

The above definition of the quasisymmetry of a tessellation (and of a simplicial map) depends apparently on the choice of a hierarchy of $\mathscr{T}$. The following corollary shows that this is not so.

Corollary 4.3. A tessellation $\mathscr{T}$ that is symmetric at non-zero vertices is quasisymmetric if and only if there is $M \geqq 0$ such that

$$
\left|\log k\left(T, T^{\prime}\right)\right| \leqq M
$$

for any two triangles $T$ and $T^{\prime}$ with three zero-angles such that $T \cap T^{\prime}$ is a common side and that both $T$ and $T^{\prime}$ group together $n$ triangles of $\mathscr{T}$ at a vertex $v \in \mathscr{T}^{0}$.

Proof. Let $\alpha: \mathscr{S} \rightarrow \mathscr{T}$ be a simplicial map, where $\mathscr{S}$ is symmetric. By Proposition 3.3 (d) there is such $\mathscr{S}$. By Proposition 3.3 (b) Corollary 4.3 is true for $\mathscr{S}$. Since we have proved that $F(\alpha)$ is quasiconformal, it follows from the quasisymmetry of the boundary extension of $F(\alpha)$ easily that Corollary 4.3 is true also for $\mathscr{T}$.

Finally, a few words on the following problem. Let $G$ be a Fuchsian group acting in $U$ and let $f: \boldsymbol{R} \rightarrow \boldsymbol{R}$ be a $G$-compatible quasisymmetric mapping. The question whether $f$ admits a $G$-compatible quasiconformal extension to $U$ is open. However, Theorem 4.2 implies a partial result:

Corollary 4.4. Let $G$ be a Fuchsian group acting on a quasisymmetric tessellation normal relative to $G$. Then any $G$-compatible quasisymmetric map $f$ admits $a$ $G$-compatible quasiconformal extension to $U$.

In particular, if $G$ is a subgroup of a finitely generated Fuchsian group $H$ such that $U / H$ is non-compact, then $G$ acts on a quasisymmetric tessellation normal relative to $G$. 
Proof. Let $G_{f}$ be the group of Möbius transformations of $U$ for which $\left\{g \mid \partial U: g \in G_{f}\right\}=f \circ G \circ f^{-1}$. Let $\mathscr{T}_{f}$ and $\alpha_{f}: \mathscr{T}_{\rightarrow} \rightarrow \mathscr{T}_{f}$ be the tessellation and the simplicial map defined in Section 2.C. $\mathscr{T}_{f}$ and $\alpha_{f}$ are uniquely determined if we demand that $\mathscr{T}_{f}$ is symmetric at non-zero vertices (this is possible since $\mathscr{T}$ is normal relative to $G$ ). Then $G_{f}$ acts on $\mathscr{T}_{f}$ and hence $G_{f}$ is Fuchsian. The quasisymmetry of $f$ implies easily that $\alpha_{f}$ is quasisymmetric. (Cf. e.g. [15, (i) in the proof of Proposition 4 p. 134] for a similar situation.) The map $F\left(\alpha_{f}\right)$ is quasiconformal and $G$-compatible since $\alpha_{f}$ is $G$-compatible. By $(\eta)$ of Section 4.C the extension of $F\left(\alpha_{f}\right)$ to $\boldsymbol{R}$ coincides with $f$ (by quasisymmetry, both $\mathscr{T}$ and $\mathscr{T}_{f}$ are tessellations of $U$ ).

The latter part of the Corollary follows from the following result.

Lemma 4.5. If $G$ is a subgroup of a finitely generated Fuchsian group $H$ such that $U / H$ is non-compact, there is a quasisymmetric tessellation $\mathscr{T}$ normal relative to $G$.

Let us assume that $H$ has a fundamental domain $D$ such that the sides $L_{i}, i \in I \cup J$, of $D$ are hyperbolic lines (if $i \in I$ ) or rays (if $i \in J$ ) such that $L_{i}$ and $L_{j}$ do not intersect except if $L_{i}$ and $L_{j}$ are rays equivalent under $H$. Let $B_{i}, i \in K$, be the components of cl $D \cap \partial U$ that are not points. Let $L_{i}, i \in K$, be the hyperbolic line with the same endpoints as $B_{i}$. Then we may assume that $L_{i}, i \in I \cup K$, are sides of $\mathscr{T}$.

$H$ has always such a fundamental domain, and if $H$ is of the second kind, we may assume in addition that if $s \in \mathscr{T}^{1}$, then at most one of the endpoints of $s$ is an elliptic or a parabolic fixpoint of $H$, and that if $\mathrm{cl} L_{i} \cap \mathrm{cl} L_{j}=X \neq \emptyset, i \neq j, i, j \in I \cup J$, then $L_{i}$ and $L_{j}$ are equivalent under $H$ and $X=\{v\}$, where $v$ is an elliptic or a parabolic fixpoint of $H$.

Proof. We assume that $H \neq\{$ id $\}$ since this case is trivial.

We prove first the existence of such a tessellation $\mathscr{S}$ for $H$ assuming the existence of a fundamental domain $D$ as described in the Lemma. It can be also assumed that if $L_{i}$ and $L_{j}$ are rays and $L_{i} \cap L_{j}=\{v\}$, then $L_{i} \cup L_{j}$ is not a hyperbolic line (i.e. $v$ is not a fixpoint for $g \in G$ of order $>2$ ). If this is the case we set $L_{i^{\prime}}=L_{i} \cup L_{j}$ and add $i^{\prime}$ to $I$ (and remove $i$ and $j$ from $J$ ) for such pairs $L_{i}$ and $L_{j}$.

Let $C_{i}, i \in K$, be the component of $U \backslash L_{i}$ for which $C_{i} \cap L_{j}=\emptyset, j \in(I \cup J \cup K) \backslash\{i\}$. Let $D^{\prime}$ be the subset of $U$ for which $\operatorname{bd}_{U} D^{\prime}=\bigcup_{i \in I U J \cup K} L_{i}$. Now we add hyperbolic lines to $D^{\prime}$ in such a way that a tessellation of $D^{\prime}$ results. This is a finite tessellation. Then we tessellate each $C_{i}$ by triangles with three zero-angles in such a way that if $T$ and $T^{\prime}$ are two adjacent triangles of this tessellation, the glide coefficient $k\left(T, T^{\prime}\right)=1$. Since $D=D^{\prime} \cup\left(\bigcup_{i \in K} C_{i}\right)$, we can extend this tessellation in a unique manner to a tessellation $\mathscr{S}$ of $U$ on which $G$ acts. It is normal relative to $H$. Since $k_{s}=1$ except if $\mathrm{cl}_{H} s \in F \subset \mathscr{S}^{1} / H$, where $F$ is finite, it is easy to see that $\mathscr{S}$ satisfies (4.5), i.e. is quasisymmetric.

$G$ acts on $\mathscr{S}$, but $\mathscr{S}$ need not be normal relative to $G$, since, if $v \in \mathscr{S}^{0} \cap U$ and if $T_{i}(v), 1 \leqq i \leqq n$, are the triangles of $\mathscr{S}$ with vertex $v,\left\{T_{i}(v)\right\}, i \leqq n$, may contain more than one non-equivalent (with respect to $G$ ) triangles. Therefore we must 
modify $\mathscr{S}$. Let

$$
U_{v}=T_{1}(v) \cup \ldots \cup T_{n}(v)
$$

We retessellate $U_{v}$ in such a way that, if $g \in G$ and $g(v)=v$, then $g$ maps this retessellation onto itself, and that in this retessellation all triangles with vertex $v$ are equivalent under $G$. Also, we may assume that if $v$ is the fixpoint of an element of $G$ of order $k \geqq 3$, then $v$ is a vertex in the retessellation. We do this for all $v \in \mathscr{S}^{0} \cap U$ taking care that a tessellation $\mathscr{T}$ on which $G$ acts results. Then $\mathscr{T}$ is normal relative to $G$.

$\mathscr{T}$ is also quasisymmetric. Since $H$ is finitely generated and $\mathscr{S}$ normal relative to $H$, the number of triangles of $\mathscr{S}$ with a vertex $v \in \mathscr{S}^{0} \cap U$ is bounded if $v$ varies in $\mathscr{S}^{0} \cap U$. Therefore the number of non-equivalent (with respect to $H$ ) retessellations of $U_{v}, v \in \mathscr{S}^{0} \cap U$, is bounded. The quasisymmetry of $\mathscr{T}$ follows from this and from the quasisymmetry of $\mathscr{S}$.

Clearly, also the second paragraph of Lemma 4.5 is true and it suffices to show the existence of such a fundamental domain $D$ as claimed in the Lemma. Unfortunately, it seems that there is no proof in the literature of the existence of such a fundamental domain although [14, Proposition p. 15] is quite close. In fact, this Proposition is all that is needed for the existence of a quasisymmetric tessellation normal relative to $G$ but later, in Theorem 5.5, we will need also the last two paragraphs of Lemma 4.5.

We construct now such a fundamental domain for $H$ as claimed in the Lemma. We assume that $H$ is of the second kind. (If $H$ is of the first kind [14, Proposition p. 15] gives the desired result.) Let $S=(\operatorname{cl} U \backslash L(H)) / H$, where $L(H) \subset \partial U$ is the set of limit points of $H$. Let $p: \operatorname{cl} U \backslash L(H) \rightarrow S$ be the canonical projection, let $x_{1}, \ldots, x_{n} \in S$ be the points such that elements of $p^{-1}\left(x_{i}\right)$ are the fixpoints of elliptic elements of $H$, and let $n_{i}$ be the ramification number at points of $p^{-1}\left(x_{i}\right)$.

It follows easily from the well-known classification theorems for surfaces of finite type that $S$ can be obtained as follows. There is a subset $D^{\prime}$ of $C \cup\{\infty\}$ such that $\operatorname{cl} D^{\prime}$ is a closed Jordan domain and $D^{\prime}$ is obtained from $\mathrm{cl} D^{\prime}$ by the removal of a finite number of points from the boundary curve. By identifying certain arcs of $\partial D^{\prime}$ we get a surface homeomorphic to $S$. Let $p^{\prime}: D^{\prime} \rightarrow S$ be the map induced by this homeomorphism. Further, let $A_{i}^{\prime}$, $i \in I$, be the arcs of $\partial D^{\prime}$ that are identified. Then each $A_{i}^{\prime}$ is a closed or half-open interval of $\partial D^{\prime}$, and we may assume that $A_{i}^{\prime} \cap A_{j}^{\prime}=\emptyset, i \neq j$, except if $A_{i}^{\prime} \cap A_{j}^{\prime}=\{v\}$, where $v \in p^{\prime-1}\left(\left\{x_{1}, \ldots, x_{n}\right\}\right)$; in this case $A_{i}^{\prime}$ and $A_{j}^{\prime}$ are closed intervals and $p^{\prime}\left(A_{i}^{\prime}\right)=p^{\prime}\left(A_{j}^{\prime}\right)$, the other endpoints of $A_{i}^{\prime}$ and $A_{j}^{\prime}$ lying in $p^{-1}(\partial S)$. If $v$ is a parabolic puncture of $S$ and $U_{v}$ a sufficiently small neighbourhood of $v$, then $p^{-1}\left(U_{v}\right)$ intersects exactly two half-open $\operatorname{arcs} A_{i}^{\prime}$ and $A_{j}^{\prime}$ such that $p^{\prime}\left(A_{i}^{\prime}\right)=p^{\prime}\left(A_{j}^{\prime}\right)$ and that the endpoints of $A_{i}^{\prime}$ and $A_{j}^{\prime}$ lie in $p^{\prime-1}(\partial S)$.

If we have found such a domain $D^{\prime}$ and such a map $p^{\prime}: D^{\prime} \rightarrow S$, we can as well assume that $p^{\prime-1}(\partial S) \subset \partial U$ and that each arc $A_{i}^{\prime}$ is a subarc of a circle $C_{i}$ that is orthogonal to $\partial U$. If $p^{\prime}\left(A_{i}^{\prime}\right)=p^{\prime}\left(A_{j}^{\prime}\right)$, we assume that $C_{i}$ and $C_{j}$ have equal diameter and there is a Möbius transformation $T_{i}$ of $U$ such that $C_{i}$ and $C_{j}$ are the isometric 
circles of $T_{i}$ and $T_{i}^{-1}=T_{j}$ and that $T_{i}\left(A_{i}^{\prime}\right)=A_{j}^{\prime}$. In case $A_{i}^{\prime} \cap A_{j}^{\prime}=\{v\}$ and $p^{\prime}(v)=$ $=x_{k}$ we assume that the order of $T_{i}$ is $n_{k}$. Let $H^{\prime}$ be the group generated by $T_{i}$, $i \in I$. Then $D^{\prime}$ is a fundamental domain for $H^{\prime}$. Thus there is a homeomorphism $f:\left(\operatorname{cl} U \backslash L\left(H^{\prime}\right)\right) / H^{\prime} \rightarrow S=(\operatorname{cl} U \backslash L(H)) / H$ such that if $f(y)=x$, then the points of $p^{\prime-1}(y)$ are fixpoints of elliptic elements of $H^{\prime}$ if and only if the points of $p^{-1}(x)$ are fixpoints for $H$ and also the orders of these elements are the same. Now it follows that there is a homeomorphism $h: \operatorname{cl} U \rightarrow \operatorname{cl} U$ such that $H^{\prime}=h^{-1} \circ H \circ h$. (This is easy to see since $L(H)$ is nowhere dense. Cf. [13, Proposition 3.5] and [14, Lemma 3].)

We can now define the fundamental domain $D$ for $H$ by setting the sides of $D$ (in $U$ ) to be $A_{i}, i \in I$, where $A_{i}$ is the hyperbolic line or ray that has endpoints $h\left(v_{1}\right)$ and $h\left(v_{2}\right) ; v_{1}$ and $v_{2}$ being the endpoints of $A_{i}^{\prime}$. It is easy to see that this defines a fundamental domain $D$ for $H$ with the desired properties.

Remarks. Although it is not known whether for every Fuchsian group $G$ any $G$-compatible quasisymmetric map $f$ admits a $G$-compatible quasiconformal extension to $U$, Lehto [11] has shown that such an extension always exists if the quasisymmetry of $f$ is sufficiently near 1 .

Also, in connection with Corollary 4.4 the following result in the same paper by Lehto is of interest. Let, if $G$ is some Fuchsian group, $Q(G)$ be the set of univalent functions $f$ defined in the lower half-plane $L$ such that $f \circ G \circ f^{-1}$ is a group of Möbius transformations of $f(L)$. Let $\Delta(G)$ consist of those elements $f \in Q(G)$ that admit a quasiconformal extension $f^{\prime}$ to $C$ in such a way that $f^{\prime} \circ G \circ f^{\prime-1}$ is a group of Möbius transformations. Then

$$
\Delta(G)=\Delta(1) \cap Q(G)
$$

if and only if every $G$-compatible quasisymmetric map admits a quasiconformal extension to $U$. (In [11] there are some restrictions on $G$ but these are unnecessary. The restrictions are used only in the footnote on p. 243 to establish that $G_{f}$ of Corollary 4.4 is discontinuous. This is true anyway, cf. e.g. the proof of Corollary 4.4 since, if $U / G$ is non-compact, $G$ always acts on a tessellation normal relative to $G$ (Proposition 3.1).)

\section{Teichmüller spaces}

A. Parametrization of quasisymmetric maps. We fix a Fuchsian group $G$ acting on a normalized tessellation $\mathscr{T}$, normal relative to $G$. We also fix a $G$-invariant hierarchy $\mathscr{H}$ of triangles of $\mathscr{T}$ which is used in the definition of the map $F(\alpha)$ induced by a simplicial map $\alpha: \mathscr{T} \rightarrow \mathscr{T}^{\prime}$. We have already defined the Teichmüller space $T(G)$ of $G$ (cf. Introduction) as a set whose elements are $G$-compatible quasisymmetric maps. We show that if $\mathscr{T}$ is quasisymmetric, we can use in the definition of $T(G)$ simplicial maps $\alpha: \mathscr{T} \rightarrow \mathscr{T}^{\prime}$ instead of quasisymmetric maps. If $\mathscr{T}$ and $\alpha$ 
are quasisymmetric, $\mathscr{T}$ and $\mathscr{T}^{\prime}$ are tessellations of $U$ (Theorem 4.2), and thus $\alpha$ induces a homeomorphism $f_{\alpha}$ of $\partial U$ for which $f_{\alpha}(v)=\alpha(v)$ if $v \in \mathscr{T}^{0}$.

Proposition 5.1. Let $G, \mathscr{T}$ and $\mathscr{H}$ be as above. If $\mathscr{T}$ is quasisymmetric, the map $\quad f_{\alpha}$ is a bijection from the set of normalized, G-compatible quasisymmetric simplicial maps $\alpha: \mathscr{T} \rightarrow \mathscr{T}^{\prime}$ onto $T(G)$. Under these circumstances $T(G)$ consists of all normalized, G-compatible quasisymmetric maps of $\boldsymbol{R}$.

Proof. Let $\alpha: \mathscr{T} \rightarrow \mathscr{T}^{\prime}$ be a normalized, $G$-compatible and quasisymmetric simplicial map. Then (Theorem 4.2) $F(\alpha): U \rightarrow U$ is $G$-compatible and quasiconformal. Since $((\zeta)$ of Section 4.C) $F(\alpha)$ has an extension to a homeomorphism of cl $U$ in such a way that $F(\alpha)(v)=\alpha(v), v \in \mathscr{T}^{0} \cap \partial U, f_{\alpha}$ is quasisymmetric and $G$-compatible. Since $f_{\alpha}$ is also normalized, $f_{\alpha} \in T(G)$.

Conversely, let $f: \boldsymbol{R} \rightarrow \boldsymbol{R}$ be normalized, $G$-compatible and quasisymmetric. Then (cf. Section 2.C) there is a unique tessellation $\mathscr{T}_{f}$ and a simplicial map $\alpha_{f}: \mathscr{T} \rightarrow \mathscr{T}_{f}$ such that $\mathscr{T}_{f}$ is normal relative to $\alpha_{f} \circ G \circ \alpha_{f}^{-1}$. It follows easily from the quasisymmetry of $f$ that $\alpha_{f}$ is quasisymmetric (cf. the proof of Corollary 4.4). Then $F(\alpha)$ is quasiconformal and thus $f \in T(G)$. Obviously $\alpha_{f}$ is also $G$-compatible and normalized. Since $\alpha \mapsto f_{\alpha}$ and $f \mapsto \alpha_{f}$ are inverse to each other, this proves our Proposition.

Thus, if $\mathscr{T}$ is quasisymmetric, we can identify $T(G)$ and the set of normalized, $G$-compatible quasisymmetric simplicial maps $\alpha: \mathscr{T} \rightarrow \mathscr{T}^{\prime}$. We do this identification in the sequel and write $\alpha \in T(G)$ for such maps $\alpha$.

In the following theorem $M(G)$ is the set of $G$-compatible complex dilatations (cf. Introduction).

Theorem 5.2. Let $G$ be a Fuchsian group acting on a quasisymmetric tessellation $\mathscr{T}$ normal relative to $G$. Then we have:

(a) The Teichmüller space $T(G)$ of $G$ is contractible.

(b) The map $\alpha \mapsto \mu(F(\alpha))$ is a real analytic section $s: T(G) \rightarrow M(G)$; i.e. $k \circ s=\mathrm{id}$ if $k: M(G) \rightarrow T(G)$ is the natural projection.

In particular, if $G$ is a subgroup of a finitely generated group $H$ such that $U / H$ is non-compact, then $G$ acts on such a tessellation $\mathscr{T}$.

Proof. Since $M(G)$ is contractible (a) follows from (b). As for (b), by Theorem 4.2 (c) it suffices to show that $k$ is real analytic if regarded as a map $M(G) \rightarrow F$, where $F$ is the $l^{\infty}$-space of sequences $\left(k_{\lambda}\right)_{\lambda \in P}$ (cf. Section 4.D). That this map is real analytic follows from Lemma of [15, Section 5].

In Corollary 4.4 we proved that if $G$ is a subgroup of a finitely generated group with non-compact quotient space, there is such a tessellation as specified by the Theorem.

In Section 4.D we defined, given a simplicial map $\alpha: \mathscr{T} \rightarrow \mathscr{T}^{\prime}$, numbers $k_{\lambda}(\alpha)$, $\lambda \in \boldsymbol{N} \times \boldsymbol{Z} \times\left(\mathscr{T}^{0} \cap \partial U\right)=P$. These numbers define $\alpha$ uniquely if it is normalized. On the other hand, the numbers $k_{\lambda}(\alpha), \lambda \in P$, are not independent. E.g. it suffices to 
know $k_{0 j v}(\alpha), j \in \boldsymbol{Z}, v \in \mathscr{T}^{0} \cap \partial U$, to determine $\alpha$, and if $\alpha$ is $G$-compatible, this gives more relations.

We determine now a subset $Q \subset P$ such that any sequence $\left(k_{\lambda}\right), \lambda \in Q$, determines a unique $G$-compatible simplicial map $\alpha: \mathscr{T} \rightarrow \mathscr{T}^{\prime}$ (not necessarily quasisymmetric) such that $k_{\lambda}=k_{\lambda}(\alpha)$ for $\lambda \in Q$. Our calculations here are quite similar to those of [15, Section 3].

We fix some $v \in \mathscr{T}^{0} \cap \partial U$ and transform the situation by a Möbius transformation of $U$ in such a way that $\alpha(v)=\infty$. Then $\alpha\left(T_{i, j-1}(v)\right) \cup \alpha\left(T_{i j}(v)\right)$ has three vertices on $\boldsymbol{R}$, let them be $a, b$ and $c$ with $a<b<c$. We have

$$
k_{i j v}(\alpha)=(c-b) /(b-a), \quad i>0, \quad j \in \boldsymbol{Z} .
$$

Let $k_{\lambda}=k_{\lambda}(\alpha), \lambda \in P$. Then a simple calculation shows

$$
k_{i, 2 j, v}=k_{i+1, j, v} \frac{1+k_{i, 2 j-1, v}^{-1}}{1+k_{i, 2 j+1, v}}
$$

for $i \geqq 1, j \in \boldsymbol{Z}$. If $i=0$, the situation is more complicated. On the one hand, a triangle $T_{0 j}(v)$ may have a non-zero angle and, on the other hand, a triangle $T_{1 j}(v)$ need not group together exactly two triangles of level 0 . However, it is easy to see that there are numbers $c_{j}, 1 \leqq c_{j} \leqq C<\infty$ for $j \in \boldsymbol{Z}$ depending on the maximal angle $\vartheta$ of $T_{0 j}(v), 0 \leqq \vartheta \leqq 120^{\circ}$, such that

$$
k_{0 j v}(\alpha)=\left(c_{j}(c-b)\right) /\left(c_{j-1}(b-a)\right),
$$

where $a<b<c$ and the line with vertices $v=\infty$ and $a$ (or $b$ or $c$ ) contains a side of $\alpha\left(T_{0, j-1}(v)\right)$ or $\alpha\left(T_{0 j}(v)\right)$. Let

$$
k_{0 j v}^{\prime}=\left(c_{j-1} / c_{j}\right) k_{0 j v}=(c-b) /(b-a) \text { for } j \in \boldsymbol{Z} .
$$

Let $m(j), j \in \boldsymbol{Z}$, be the numbers such that $T_{1 j}(v)$ groups together triangles $T_{0 m(j)}(v), T_{0, m(j)+1}(v), \ldots, T_{0, m(j+1)-1}(v)$. Now a similar calculation as in (5.2) shows

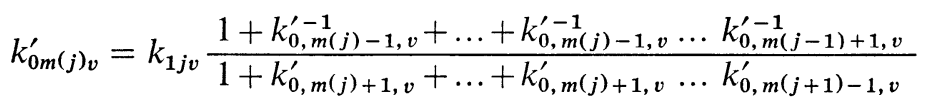

for $j \in \boldsymbol{Z}$. A repeated application of (5.2) gives

$$
k_{i, 2^{n} j, v}=k_{i+n, j, v} \frac{1+k_{i+n-1,2 j-1, v}^{-1}}{1+k_{i+n-1,2 j+1, v}} \ldots \frac{1+k_{i, 2^{n} j-1, v}^{-1}}{1+k_{i, 2^{n} j+1, v}}
$$

if $i \geqq 1$ and $j \in \boldsymbol{Z}$.

Let $s \in \mathscr{T}^{1}$ be a hyperbolic line with endpoints $v$ and $v^{\prime}$. Let $j(s)$ and $j^{\prime}(s) \in \boldsymbol{Z}$ be the numbers such that $s=s_{0 j(s)}(v)=s_{0 j^{\prime}(s)}\left(v^{\prime}\right)$. Then we have

$$
k_{0 j(s) v}=k_{0 j^{\prime}(s) v^{\prime}}
$$

for every such side $s$. If $s \in \mathscr{T}^{1}$ is a hyperbolic ray with vertex $v \in \mathscr{T}^{0} \cap \partial U$, the 
normality of $\alpha \circ G \circ \alpha^{-1}$ relative to $\mathscr{T}^{\prime}$ gives

$$
k_{0 j(s) v}=1
$$

if $j(s) \in \boldsymbol{Z}$ is the number for which $s=s_{0 j(s)}(v)$.

Next we seek for the relations that derive from the $G$-compatibility of $\alpha$. If $\alpha$ is $G$-compatible, we must have

$$
k_{i j g(v)}=k_{i j v} \text { for }(i, j, v) \in P \text { and } g \in G
$$

since the hierarchy $\mathscr{H}$ is $G$-compatible. If $\alpha(v)$ is a fixpoint of $\alpha \circ G \circ \alpha^{-1}$, we have

$$
k_{i j v}=k_{i, j+n, v} \text { for } i \geqq 0 \text { and } j \in Z
$$

provided there is $g \in G$ with fixpoint $v$ such that $g\left(s_{i j}(v)\right)=s_{i, j+n}(v)$. In case $\alpha(v)$ is a parabolic fixpoint of $\alpha \circ G \circ \alpha^{-1}$ we have also

$$
k_{i j v}=1 \text { if } i \geqq i_{0} \text { and } j \in Z \text {, }
$$

where $i_{0} \geqq 0$ is the smallest number $i$ for which all the sides of $\left\{s_{i j}(v): j \in Z\right\}$, oriented with $v$ as the positive endpoint, are equivalent under $G$. It is easy to see that (5.9) is a consequence of (5.4) and (5.8) if $\sup _{\lambda \in \boldsymbol{P}}\left|\log k_{\lambda}\right|<\infty$; i.e. if $\alpha$ is quasisymmetric.

Let us suppose that numbers $k_{\lambda}>0, \lambda \in P$, satisfy (5.2)-(5.8). Then we assign for each $s \in \mathscr{T}^{1}$ that is a hyperbolic line the number $k_{s}=k_{0 j v}$ if $v$ is an endpoint of $s$ and $s_{0 j}(v)=s$. By (5.5) $k_{s}$ does not depend on the chosen endpoint of $s$. Then (Section 3.C) there is a unique, normalized tessellation $\mathscr{T}^{\prime}$, symmetric at non-zero vertices, and a unique normalized simplicial map $\alpha: \mathscr{T} \rightarrow \mathscr{T}^{\prime}$ such that these numbers $k_{s}$ are the glide coefficients of $\mathscr{T}^{\prime}$. It is obvious, since $k_{s}$ 's satisfy (5.2)-(5.8), that we have then $k_{\lambda}(\alpha)=k_{\lambda}$ for $\lambda \in P$ and that $\alpha$ is $G$-compatible. We paraphrase this in the following

Lemma 5.3. Let the numbers $k_{\lambda}>0, \lambda \in P$, satisfy (5.2)-(5.8). Then there is a unique normalized G-compatible simplicial map $\alpha: \mathscr{T} \rightarrow \mathscr{T}^{\prime}$ such that $k_{\lambda}(\alpha)=k_{\lambda}$, $\lambda \in P$. If $\sup _{\lambda \in \boldsymbol{P}}\left|\log k_{\lambda}\right|<\infty$, then $\alpha$ is quasisymmetric and the numbers $k_{\lambda}, \lambda \in P$, satisfy also (5.9).

To proceed we must make additional assumptions on the hierarchy $\mathscr{H}$. We assume that the $G$-invariant hierarchy $\mathscr{H}$ satisfies also (f) below, in addition to the conditions (a)-(e) of Section 3.B. This condition is motivated by the construction in Theorem 5.5.

(f) There is a partition $I_{i}, i \geqq 0$, of $\mathscr{T}^{0}$ with the following properties:

(1) If $v \in \mathscr{T}^{0}$ is a fixpoint of $G \backslash\{\mathrm{id}\}$, then $v \in I_{0}$. If $s \in \mathscr{T}^{1}$, at most one endpoint of $s$ is in $I_{0}$.

(2) If $v \in I_{1}$, then the number of sides $s \in \mathscr{T}^{1}$ with vertex $v$ such that the other vertex is in $I_{0} \cup I_{1}$ does not exceed a constant $N_{1}$. If $s_{0 j}(v)$ has endpoint $v^{\prime}(\neq v)$ in $I_{0} \cup I_{1}$, then $s_{0 j}(v) \notin\left\{s_{1 j}(v): j \in Z\right\}$. 
(3) If $v \in I_{k}, k \geqq 2$, then each triangle of level 1 groups together exactly two triangles of level 0 . If $v^{\prime}$ is the other endpoint of $s_{0 j}(v)$, then $v^{\prime} \in I_{k^{\prime}}$, where $k^{\prime}>k$ except if $j=0$ or $j=1$ when $k^{\prime}<k$. In addition, $s_{02}(v)=s_{00}\left(v^{\prime}\right)$.

We shall later show that if $G$ is a subgroup of a finitely generated group of the second kind, then there is a quasisymmetric tessellation normal relative to $G$ with a $G$-invariant hierarchy that satisfies (a)-(f).

If the hierarchy satisfies also (f) we can find a basis $k_{\lambda}, \lambda \in Q$, for $k_{\lambda}$ 's as follows. Let $F=\left\{v_{1}, v_{2}, \ldots\right\}$ contain exactly one element from each orbit $G v, v \in \mathscr{T}^{0} \cap \partial U$. For elements of $\mathscr{T}^{0} \cap \partial U$ we define an order by setting $v<v^{\prime}$ if $\operatorname{cl} v=\operatorname{cl} v_{n}$ and $\operatorname{cl} v^{\prime}=\operatorname{cl} v_{n^{\prime}}$ (in $\mathscr{T} / G$ ) and $n<n^{\prime}$. We define a subset $Q \subset P$ by (i)-(iii).

(i) Let $v \in F \cap I_{k}, k \geqq 2$. Then $(i, j, v) \in Q$ if $i \geqq 0$ and $j \notin 2 Z$ with the exception of $(0,1, v) \notin Q$.

(ii) Let $v \in F \cap\left(I_{0} \cup I_{1}\right)$ and assume that $v$ is not a fixpoint of $G \backslash\{$ id $\}$. Let $i \geqq 1$; then $(i, j, v) \in Q$ if $j \notin 2 Z$ and, in addition, $(1,0, v) \in Q$. Let $v \in I_{0}$; then $(0, j, v) \in Q$ if $s_{0 j}(v) \notin\left\{s_{1 k}(v): k \in Z\right\}$. Let $v \in I_{1}$; then $(0, j, v) \in Q$ if $s_{0 j}(v) \notin\left\{s_{1 k}(v): k \in \boldsymbol{Z}\right\}$ and if the other endpoint $v^{\prime}$ of $s_{0 j}(v)$ is in $I_{k}, k \geqq 2$, or in $I_{1}$ and $v^{\prime}>v$ or $\mathrm{cl} v=\mathrm{cl} v^{\prime}$ (in $\left.\mathscr{T}^{0} / G\right)$ and $s_{0 j}(v)=s_{0 j^{\prime}}\left(v^{\prime}\right)$ with $j<j^{\prime}$.

(iii) If $v$ is a fixpoint of $G \backslash\{\mathrm{id}\}$ (now necessarily $\left.v \in I_{0}\right)$, then $(i, j, v) \in Q$ if $(i, j, v)$ satisfies (ii) except that $(1,0, v) \notin Q$, and, in addition, $i<M$ and $0<j<m$. Here $M>0$ is the smallest number for which all the sides of $\left\{s_{M k}(v): k \in Z\right\}$ oriented with $v$ as the positive endpoint are equivalent under $G$ and $m>0$ is the smallest number for which there is $g \in G$ with $g(v)=v$ such that $g\left(s_{i 0}(v)\right)=s_{i m}(v)$.

Let $k_{\lambda}, \lambda \in Q$, be a sequence of positive real numbers. We determine $k_{\lambda}, \lambda \in P \backslash Q$, in such a way that $k_{\lambda}, \lambda \in P$, satisfies (5.2)-(5.9). Let $v \in I_{0} \cap F$. Then, in view of (ii) and (iii), (5.3), (5.4), (5.7), and, if $v$ is a fixpoint of $G \backslash\{$ id\}, (5.8) and (5.9) determine all the remaining $k_{i j v}$ 's. Let then $v \in I_{1} \cap F$ and assume that we know all $k_{i j v^{\prime}}$ 's if $v^{\prime} \in I_{0}$ or $v^{\prime} \in I_{1}$ and $v^{\prime}<v$. Again, in view of (ii), eq. (5.3), (5.4), (5.6) and (5.7) determine all $k_{i j v}$ 's. We continue in this way. We assume that we know $k_{i j v}$ if $v \in I_{k}$ with $k<n$ and determine all the numbers $k_{i j v}, v \in I_{n}$, from (5.3), (5.4), (5.6) and (5.7). Clearly, in this manner all the numbers $k_{\lambda}, \lambda \in P$, are determined uniquely and they satisfy (5.2)-(5.9). Notice that if $v \in \mathscr{T}^{0} \cap \partial U$ is a fixpoint of $G \backslash\{$ id $\}$, then $\alpha(v)$ is a parabolic fixpoint of $\alpha \circ G \circ \alpha^{-1}$.

Thus we have proved:

Lemma 5.4. Let $k_{\lambda}, \lambda \in Q$, be positive real numbers. Then there is a unique normalized $G$-compatible simplicial map $\alpha: \mathscr{T} \rightarrow \mathscr{T}^{\prime}$ such that $k_{\lambda}=k_{\lambda}(\alpha), \lambda \in Q$, for which $\alpha(v)$ is a parabolic fixpoint of $\alpha \circ G \circ \alpha^{-1}$ whenever $v \in \partial U$ is a fixpoint of some element of $G \backslash\{\mathrm{id}\}$.

B. Embedding into a Banach space. In this section we show how to embed all normalized quasisymmetric and $G$-compatible simplicial maps of a quasisymmetric 
tessellation $\mathscr{T}$ with a $G$-invariant hierarchy $\mathscr{H}$ satisfying (a)-(f) into a Banach space. In the preceding section we did not assume that $\mathscr{T}$ was quasisymmetric; from now on we make this assumption. Thus $\mathscr{T}$ is a tessellation of $U$ (Theorem 4.2), and if $v \in \mathscr{T}^{0} \cap \partial U$ is a fixpoint of $G \backslash\{$ id $\}, v$ is a parabolic fixpoint of $G$.

By Lemma 5.3 we can identify the set of normalized, quasisymmetric $G$-compatible simplicial maps $\alpha$ of $\mathscr{T}$ with sequences $\left(k_{\lambda}\right)_{\lambda \in P}$ of positive real numbers that satisfy relations (5.2)-(5.9) with $\sup _{\lambda \in P}\left|\log k_{\lambda}\right|<\infty$. We do this identification and mean the map $\alpha$ when we say the map $\left(k_{\lambda}(\alpha)\right)_{\lambda \in P}=\left(k_{\lambda}\right)_{\lambda \in P}$. Since, by Lemma 5.4 , it suffices to know $k_{\lambda}$ for $\lambda \in Q$, we can also say the map $\left(k_{\lambda}\right)_{\lambda \in Q}$. Then it is understood that the numbers $k_{\lambda}, \lambda \in P \backslash Q$, are determined from relations (5.2)-(5.9).

Given a simplicial map $\left(k_{\lambda}\right)_{\lambda \in P}$, we define the numbers $\left(h_{\lambda}\right)_{\lambda \in Q}$ by

$$
\begin{aligned}
h_{i j v} & =\log k_{i j v} \quad \text { if } \quad(i, j, v) \in Q \quad \text { and } i=0 \quad \text { or } \quad(i, j, v)=(1,0, v), \\
h_{i j v} & =\log \left(1+k_{i j v}\right) \quad \text { if } i \geqq 1, \quad(i, j, v) \in Q \quad \text { and } j-1 \in 4 Z, \\
& =\log \left(1+k_{i j v}^{-1}\right) \quad \text { if } i \geqq 1, \quad(i, j, v) \in Q \quad \text { and } j+1 \in 4 Z .
\end{aligned}
$$

With the numbers $\left(h_{\lambda}\right)_{\lambda \in Q}$, equation (5.4) assumes form

$$
\begin{aligned}
\log k_{i, 2^{n} j, v} & =\log k_{i+n, j, v} \\
& +\log \left(1+k_{i+n-1,2 j-1, v}^{-1}\right)-\log \left(1+k_{i+n-1,2 j+1, v}\right) \\
& +\sum_{l=2}^{n}\left(h_{i+n-l, 2 l_{j}-1, v}-h_{i+n-l, 2 l_{j}+1, v}\right)
\end{aligned}
$$

for $i \geqq 1, j \in \boldsymbol{Z}, v \in F, n \geqq 0$. If $v$ is a parabolic fixpoint of $G$, we assume also that all the indices appearing in the $h$ 's are in $Q$. If $j=0$, we need (5.11) in a slightly different form:

$$
\log k_{i+n, 0, v}=\log k_{i 0 v}-\sum_{l=0}^{n-1}\left(h_{i+l,-1, v}-h_{i+l, 1, v}\right)
$$

for $i \geqq 1, n \geqq 0$, and $v \in F$. If $v$ is a parabolic fixpoint of $G$, we set $h_{k,-1, v}=h_{k, m-1, v}$, where $m>0$ is the smallest number for which $g\left(s_{k 0}(v)\right)=s_{k m}(v)$ for some $g \in G$ with $g(v)=v$. In this case we also assume that $i+n \leqq N$, where $N \geqq 0$ is the smallest number for which all the sides of $\left\{s_{N j}(v): j \in Z\right\}$, oriented with $v$ as the positive endpoint, are equivalent under $G$.

In view of (5.11) and (5.12) it is reasonable to consider a Banach space $E$ whose elements are sequences $\left(h_{\lambda}\right)_{\lambda \in Q}$ of real numbers such that

$$
\begin{aligned}
& m_{1}=\sup _{\lambda \in Q}\left|h_{\lambda}\right|, \\
& m_{2}=\sup \left\{\left|\sum_{l=1}^{n}\left(h_{i+n-l, 22_{j}-1, v}-h_{i+n-l, 2 l}+1, v\right)\right|:(i+n, j, v) \in Q, i \geqq 1, j \neq 0, n \geqq 0\right\}, \\
& m_{3}=\sup \left\{\left|\sum_{l=1}^{n}\left(h_{1+l,-1, v}-h_{1+l, 1, v}\right)\right|: n \geqq 0,(1+n, 1, v) \in Q\right\}
\end{aligned}
$$


are finite. For such sequences we define the norm to be

$$
\left\|\left(h_{\lambda}\right)_{\lambda \in Q}\right\|_{E}=\max \left\{m_{1}, m_{2}, m_{3}\right\} .
$$

Finally, let $E_{0}$ be the open and convex subset of $E$ consisting of those sequences $\left(h_{\lambda}\right)_{\lambda \in Q}$ for which

$$
\inf \left\{h_{i j v}:(i, j, v) \in Q \text { and } i \geqq 1 \text { and }(i, j) \neq(1,0)\right\}>0 \text {. }
$$

By Proposition 5.1, we have identified the Teichmüller space $T(G)$ of $G$ with the set of normalized, quasisymmetric simplicial $G$-compatible maps of $\mathscr{T}$ and this we have identified with sequences $\left(k_{\lambda}\right)_{\lambda \in P}$ which satisfy (5.2)-(5.9) with $\sup _{\lambda \in P}\left|\log k_{\lambda}\right|<\infty$. Let $\varphi: T(G) \rightarrow E$ be the map $\left(k_{\lambda}\right)_{\lambda \in P} \mapsto\left(h_{\lambda}\right)_{\lambda \in Q}$ defined by (5.10).

Theorem 5.5. Let $G$ act on a quasisymmetric tessellation $\mathscr{T}$, normal relative to $G$ that has a $G$-invariant hierarchy $\mathscr{H}$ satisfying (a)-(f). Then the image of the map $\varphi: T(G) \rightarrow E$ is $E_{0}$ and $\varphi$ is a real analytic isomorphism $T(G) \rightarrow E_{0} . E_{0}$ is an open and convex subset of $E$ and it is homeomorphic to $E$. $E$ is isomorphic to $l^{\infty}$ as a real Banach space.

In particular, if $G$ is a subgroup of a finitely generated group of the second kind, the assumptions of this theorem are satisfied.

Proof. Clearly, if $\alpha \in T(G), \varphi(\alpha) \in E_{0}$. Lemma 5.4 implies that $\varphi$ is an injection. To show that $\varphi$ is a real analytic isomorphism of $T(G)$ onto $E_{0}$ it suffices to show:

(i) The map $\varphi \circ k: M(G) \rightarrow E$ is real analytic, where $M(G)$ is as in Introduction and $k: M(G) \rightarrow T(G)$ is the canonical projection.

(ii) $E_{0} \subset \operatorname{im} \varphi$ and $h \mapsto \mu\left(F\left(\varphi^{-1}(h)\right)\right)$ is a real analytic map $E_{0} \rightarrow M(G)$, where $F\left(\varphi^{-1}(h)\right): U \rightarrow U$ is the map defined in Section 4.C.

It follows from (i) that $\varphi$ is real analytic and (ii) implies that $\varphi^{-1}$ is real analytic.

We prove (i). Let $F$ be the $l^{\infty}$-space of sequences $\left(k_{\lambda}\right)_{\lambda \in P}$ of real numbers. Then $T(G) \subset F$, and let $k(\mu)=\left(k_{\lambda}(\mu)\right)_{\lambda \in P}$ for $\mu \in M(G)$. If $\lambda=(i, j, v)$, we obtain $k_{\lambda}(\mu)$ as follows. Let $f$ be a quasiconformal self-map of $\operatorname{cl} U$ with dilatation $\mu$ such that $f(v)=\infty$. Then

$$
k_{\lambda}(\mu)=\frac{f(c)-f(b)}{f(b)-f(a)},
$$

where $a, b, c$ and $v$ are the vertices of the triangles $T_{i j}(v)$ and $T_{i, j-1}(v)$ of $\mathscr{T}$ and $f(a)<f(b)<f(c)$ (cf. eq. (5.1)). Lemma of [15, Section 5 p. 139] implies that $k$ is real analytic if regarded as a map $M(G) \rightarrow F$. Now a glance at (5.10)-(5.14) shows that $\varphi \circ k$ is real analytic.

We then prove (ii). Let $\left(h_{\lambda}\right)_{\lambda \in Q}=h \in E_{0}$. Define $k_{\lambda}$ for $\lambda \in Q$ in such a way that (5.10) is true. By Lemma 5.4 there is a unique normalized, simplicial map $\alpha: \mathscr{T} \rightarrow \mathscr{T}^{\prime}$ such that $k_{\lambda}(\alpha)=k_{\lambda}$ for $\lambda \in Q$ and that $\alpha(v)$ is a parabolic vertex of $\mathscr{T}^{\prime}$ whenever $v$ is a parabolic fixpoint of $\mathscr{T}$. Let $k_{\lambda}=k_{\lambda}(\alpha)$ for all $\lambda \in P$. We must show that 
$\sup _{\lambda \in P}\left|\log k_{\lambda}\right|<\infty$. On the basis of (5.11)-(5.13), (ii), (iii), (c) and (2) of (H) in the definition of $\mathscr{H}$ it is to easy see that there is $M_{1}>0$ such that

$$
\left|\log k_{i j v}\right|<M_{1} \text { for }(i, j, v) \in P \text { and } v \in I_{0} \cup I_{1} \text {. }
$$

Eq. (5.11) and (i) in the definition of $Q$ imply that we may assume $M_{1}$ to be chosen in such a way that (5.16) is true also if $v \in I_{k}, k \geqq 2$, if $j \neq 0,(i, j) \neq(0,2)$ and $(i, j) \neq$ $\neq(0,1)$. Then, by (3) in the requirement (f) for $\mathscr{H}$ (in Section 5.A), $s_{01}(v)=s_{0 j^{\prime}}\left(v^{\prime}\right)$, where $v^{\prime} \in I_{k^{\prime}}$ with $k^{\prime}<k$ and, if $k^{\prime}>2, j^{\prime} \neq 0,1$ or 2 . Therefore (5.16) is true also for $k_{01 v}$ for all $v$. The number $k_{02 v}$ is calculated from

$$
k_{02 v}^{\prime}=k_{11 v} \frac{1+k_{01 v}^{\prime-1}}{1+k_{03 v}^{\prime}}
$$

(cf. (5.3), $k_{0 j v}^{\prime}=\left(c_{j-1} / c_{j}\right) k_{0 j v}$ is as in (5.3)). Since we now know that (5.16) is true for $k_{11 v}, k_{03 v}$ and $k_{01 v}$, there is $M_{2}>0$ such that

$$
\left|\log k_{02 v}\right|<M_{2} \text { for all } v \in \mathscr{T}^{0} \cap \partial U \text {. }
$$

Finally, requirement (3) of (f) together with (5.17), (5.16), (5.12) and (5.2) implies that there is $M_{3}$ such that

$$
\left|\log k_{i 0 v}\right|<M_{3} \text { for all } i \geqq 0 \text { and } v \in \mathscr{T}^{0} \cap \partial U .
$$

Thus we have shown that $\sup _{\lambda \in P}\left|\log k_{\lambda}\right|<\infty$.

To prove that $h \mapsto \mu\left(F\left(\varphi^{-1}(h)\right)\right)$ is real analytic, notice that by Theorem 4.2 it suffices to show that $\varphi^{-1}$ is real analytic if regarded as a map $E_{0} \rightarrow F$. This can be seen as follows. We have used in calculating $k_{\lambda}, \lambda \in P \backslash Q,(5.7),(5.11),(5.12)$ and also (5.3) to calculate $k_{0 j v}$ if $(0, j, v) \notin Q$. In (5.11) and (5.12) there is a part that depends linearly on the $h_{\lambda}$ 's and that part is bounded by the norm condition (5.14). The non-linear part depends real-analytically on a finite number of $h_{\lambda}$ 's and there is only a finite number of essentially different non-linear parts, i.e. such that cannot be obtained from others by a permutation of the $h_{\lambda}$ 's. A similar remark applies to (5.3). Now a similar argument as in the proof that $\sup _{\lambda \in \boldsymbol{P}}\left|\log k_{\lambda}\right|<\infty$ shows that $\varphi^{-1}$ is real analytic.

$E_{0}$ is clearly open and convex and a homeomorphism $E_{0} \rightarrow E$ is constructed as in Corollary in [15, Section 3 p. 132]. (Note that the definition of $E_{0}$ is slightly different in [15, eq. (6) p. 131].)

To show that $E$ is isomorphic to $l^{\infty}$ we construct a different "basis" for $E$. Let $Q_{1}=\{(i, j, v) \in Q: i \geqq 1$ and $j+1 \in 4 Z\}$. If $\left(h_{\lambda}\right)_{\lambda \in Q} \in E$ let $h_{\lambda}^{\prime}=h_{\lambda}$ if $\lambda \notin Q_{1}$ and if $(i, j, v) \in Q_{1}$ let, if $k=j+1$,

$$
h_{i j v}^{\prime}=\sum_{l=0}^{i-1}\left(h_{i-l, 2 l k-1, v}-h_{i-l, 2 l} k+1, v\right)
$$

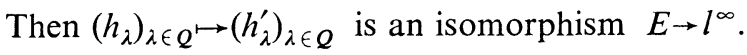


Finally, we show that a subgroup $G$ of a finitely generated Fuchsian group $H$ of the second kind acts on a tessellation $\mathscr{T}$ with a hierarchy $\mathscr{H}$ that satisfies the conditions of the Theorem.

Let $D$ be such a fundamental domain for $H$ as specified in Lemma 4.5 and let $\mathscr{T}$ be the tessellation constructed in Lemma 4.5 for $G$ by means of $D$. We have already shown that $\mathscr{T}$ is quasisymmetric. Therefore it suffices to show that there is a hierarchy $\mathscr{H}$ of $\mathscr{T}$ that satisfies also (f).

Let $L_{i}, i \in I \cup J \cup K$, be as in Lemma 4.5. Let $I_{0} \subset \mathscr{T}^{0}$ consist of $v \in \mathscr{T}^{0}$ that are elliptic fixpoints for $G$ or parabolic fixpoints for $H$. (Notice that if $v$ is a fixpoint for $H \backslash\{$ id $\}$, it need not be a fixpoint for $G \backslash\{\mathrm{id}\}$ and then, if $v \in U, v \notin \mathscr{T}^{0}$.) Let $I_{1}$ consist of those $v \in \mathscr{T}^{0}$ that are endpoints of $h\left(L_{i}\right), i \in K, h \in H$. If $k>1$, an element $v$ of $\mathscr{T}^{0} \backslash\left(I_{0} \cup I_{1}\right)$ is in $I_{k}$ if and only if there is a sequence $T_{1}, \ldots, T_{k-1}$ of triangles of $\mathscr{T}$ such that: (i) $T_{i} \cap T_{i+1}=$ a common side, $i<k-1$; (ii) $T_{i} \neq T_{j}$ if $i \neq j$; (iii) each $T_{i} \subset \mathrm{cl} C, i \leqq k-1$, where $C$ is a component of $U \backslash\left(\cup\left\{h\left(L_{i}\right): h \in H\right.\right.$ and $i \in I \cup J \cup K\}$ such that $\mathrm{bd}_{U} C=h\left(L_{i}\right) \subset T_{1}$ for some $h \in H$ and $i \in K$; (iv) $v \in T_{i}$ if and only if $i=k-1$.

It is easy to see that $I_{k}, k \geqq 0$, is a partition of $\mathscr{T}^{0}$. If $v \in I_{k}, k \geqq 2$, there is a unique way of defining the triangles $T_{i j}(v), i \geqq 0, j \in \boldsymbol{Z}$, in such a way that (3) of (f) is true. Notice that $T_{i j}(v)$ become defined in a $G$-compatible manner. If $v \in I_{0}$, we can define the triangles $T_{i j}(v)$ arbitrarily, taking only care that the result is $G$-compatible. Condition (1) of (f) is automatically true.

Let then $v \in I_{1}$. This is the most complicated case. We show first that there is such $N_{1}$ as specified in (2) of (f). Let $D^{\prime}$ be the closed domain of $U$ whose boundary is $\cup L_{i}, i \in I \cup J \cup K$. Let

$$
D^{\prime \prime}=\cup\left\{h\left(D^{\prime}\right): h \in H \text { is elliptic and } h\left(D^{\prime}\right) \cap D^{\prime} \neq \emptyset\right\} .
$$

Then $\operatorname{bd}_{U} D^{\prime \prime}$ consists of a finite number of hyperbolic lines that are of the form $h\left(L_{i}\right), h \in H$ and $i \in I \cup K$. Each line $L_{i} \subset \mathrm{bd}_{U} D^{\prime \prime}, i \in K$, and cl $D^{\prime \prime}$ (closure in $C \cup\{\infty\}$ ) is obtained from $D^{\prime \prime}$ by the addition of the endpoints of the sides. Thus each component of $\operatorname{bd}_{U} D^{\prime \prime}$ is a side of $\mathscr{T}$. If $v \in I_{1}$, then $v$ is an endpoint of a side of $\mathscr{T}$ of form $h\left(L_{i}\right), h \in H$ and $i \in K$. Therefore $v \in \mathrm{cl} h\left(D^{\prime \prime}\right)$ for some $h \in H$. If $s \in \mathscr{T}^{1}$ has endpoints $v$ and $v^{\prime}$, where also $v^{\prime} \in I_{0} \cup I_{1}$, then we must have $s \subset h\left(D^{\prime \prime}\right)$. Since $D^{\prime \prime}$ contains only a finite number of vertices of $\mathscr{T}$, there is such $N_{1}$ as claimed in (2) of (f). Now it is easy to see that we can define the triangles $T_{i j}(v)$ in such a way that (2) of (f) is true and that a $G$-compatible hierarchy $\mathscr{H}$ results.

Remark 1. If we use the "basis" $h_{\lambda}^{\prime}, \lambda \in Q$, constructed above for $E$ to show that $E$ is isomorphic to $l^{\infty}$, we get a simpler expression for the norm of $E$ (the supremum norm) but the price to be paid is that the image of $T(G)$ is more complicated.

Remark 2. Our method applies also if $G$ is a finitely generated group of the first kind such that $U / G$ is non-compact. In fact, it seems that in this manner a 
particularly simple set of "moduli" for $T(G)$ results. In this case it is better to parametrize $T(G)$ by the use of the glide coefficients $k_{s}, s \in F$, where $F$ is some set that contains exactly one point from each orbit $G \mathscr{T}^{1}$. If $s$ has an endpoint $v$ that is an elliptic fixpoint for $G$, then we must have $k_{s}=1$. This is true also if $v$ is a parabolic fixpoint for $G$ and there is only one triangle of $\mathscr{T} / G$ with vertex $\mathrm{cl}_{G} v$. For each remaining parabolic fixpoint $v$ of $F$ we have a relation since we must choose the numbers $k_{s}$ in such a way that a parabolic fixpoint results. Let $k_{1}, \ldots, k_{n}$ be the glide coefficients of the sides $s_{1}, \ldots, s_{n}$ of $\mathscr{T}^{1} / G$ with vertex $\operatorname{cl}_{G} v$, where $s_{1}, \ldots, s_{n}$ are in cyclic order. Note that we may have that $s_{i}=s_{j}$ even if $i \neq j$ since the endpoints of a side of $\mathscr{T}^{1}$ may be equivalent under $G$. Then an equation similar to (5.3) (set $k_{1 j v}=1$ in (5.3)) can be used to obtain the condition for $v$ to be parabolic. We have, after some manipulation,

$$
1=k_{1}^{\prime} \ldots k_{n}^{\prime},
$$

where $k_{i}^{\prime}=d_{i} k_{1}$ and $d_{i}$ is a constant $\left(=c_{j-1} / c_{j}\right.$ of (5.3)) that depends on the angles of the triangles with side $s_{i}$ (if they have three zero-angles then $d_{i}=1$ ). Thus, if $h_{i}=\log k_{i}^{\prime}$, then for each parabolic puncture of $U / G$ we have a relation of the form

$$
h_{1}+\ldots+h_{n}=0 \text {. }
$$

Hence we can choose a subset $F^{\prime} \subset F$ in such a way that $h_{s}, s \in F^{\prime}$, can be determined freely and that they determine the other $h$ 's uniquely. Consequently $T(G)$ is equivalent as a real analytic manifold to a finite dimensional euclidean space.

\section{References}

[1] Bers, L.: On moduli of Riemann surfaces. - Lecture notes, Eidgenössische Technische Hochschule, Zürich, 1964, (mimeographed).

[2] Bers, L.: Universal Teichmüller space. - Analytic methods in mathematical physics, edited by R. P. Gilbert and R. G. Newton, Gordon and Breach, New York-LondonParis, 1968, 65-83.

[3] Carleson, L.: The extension problem for quasiconformal mappings. - Contributions to Analysis, edited by L. V. Ahlfors et al., Academic Press, New York-London, 1974, $39-47$.

[4] Earle, C.: The contractibility of certain Teichmüller spaces. - Bull. Amer. Math. Soc. 73, 1967, 434-437.

[5] Earle, C.: On quasiconformal extensions of the Beurling-Ahlfors type. - Contributions to Analysis, edited by L. V. Ahlfors et al., Academic Press, New York-London, 1974, 99-105.

[6] Earle, C., and J. Eells: On the differential geometry of Teichmüller spaces. - J. Analyse Math. 19, 1967, 35-52.

[7] KeEN, L.: Intrinsic moduli on Riemann surfaces. - Ann. of Math. 84, 1966, 404-420.

[8] KeEn, L.: On Fricke moduli. - Advances in the theory of Riemann surfaces, edited by L. V. Ahlfors et al., Annals of Mathematics Studies, No. 66, Princeton University Press, Princeton, N. J., 1971, 205-224.

[9] Keen, L.: A correction to "On Fricke moduli". - Proc. Amer. Math. Soc. 40, 1973, 60-62. 
[10] KeEN, L.: On infinitely generated Fuchsian groups. - J. Indian Math. Soc. 35, 1971, 67-85.

[11] LeHto, O.: Group isomorphisms induced by quasiconformal mappings. - Contributions to Analysis, edited by L. V. Ahlfors et al., Academic Press, New York-London, 1974, $241-244$.

[12] Pexcszyński, A.: On the isomorphism of the spaces $m$ and $M$. - Bull. Acad. Polon. Sci. Sér. Sci. Math. Astronom. Phys. 6, 1958, 695-696.

[13] Tukia, P.: On discrete groups of the unit disk and their isomorphisms. - Ann. Acad. Sci. Fenn. Ser. A I 504, 1972, 1-45.

[14] Tukia, P.: Extension of boundary homeomorphisms of discrete groups of the unit disk. - Ibid. $548,1973,1-16$.

[15] Tukia, P.: The space of quasisymmetric mappings. - Math. Scand. 40, 1977, 127-142.

\author{
University of Helsinki \\ Department of Mathematics \\ SF-00100 Helsinki 10 \\ Finland
}

Received 2 November 1977 